\title{
Anonymous Procedures for Condorcet's Model: Robustness, Nonmonotonicity, and Optimality*
}

\author{
Michael Suk-Young Chwe \\ Department of Political Science, UCLA, Los Angeles, CA 90095-1472, USA \\ Email:michael@chme.net; mmm.chme.net
}

\begin{abstract}
In Condorcet's model of information aggregation, a group of people decides among two alternatives $a$ and $b$, with each person getting an independent bit of evidence about which alternative is objectively superior. I consider anonymous procedures, in which the group's decision depends only on the number of people who report $a$ or $b$, not their identities. A procedure is called incentive compatible for a person if she wants to report truthfully given that others report truthfully. I show that if an anonymous procedure is incentive compatible for both a person who is significantly biased toward $a$ and a person who is significantly biased toward $b$, then it is incentive compatible for any person, regardless of his preferences and prior beliefs; also, if it is not trivial, it must be nonmonotonic, with an additional report for $a$ sometimes decreasing the probability the group chooses $a$. I define the "supermajority penalty" (SP) procedure and show that when there are significant biases in both directions, the SP procedure is the optimal anonymous incentive compatible procedure from the point of view of an unbiased person.
\end{abstract}

People often make important decisions collectively because they believe that by combining their judgment, they are more likely to make the correct decision. Examples include juries who collectively decide whether to find a person guilty or innocent, journal editors and referees who collectively decide whether to accept a manuscript, and consulting

* This paper arose out of conversations with Leeat Yariv and has benefited from suggestions by Jeff Lewis, Balázs Szentes, Eddie Dekel, César Martinelli, participants at the 2009 Wallis Conference on Political Economy at the University of Rochester, and anonymous referees.

MS submitted 3 October 2008; final version received 10 November 2009

ISSN 1554-0626; DOI 10.1561/100.00008077

(C) 2010 M. S.-Y. Chwe 
doctors who collectively decide how to treat a patient. In these situations, people's prior beliefs or inclinations might differ, but their interests do not fundamentally conflict; if it were known for certain whether a person was guilty or innocent, or what the best treatment for a patient would be, then all would agree.

A mathematical representation of this kind of collective decision-making was introduced by Condorcet (1785). In his model, there are a group of $n$ people who have to decide among two alternatives $a$ and $b$, and each gets an independent bit of evidence about which alternative is objectively best. Each person's evidence either favors $a$ or $b$, and each person's evidence is assumed to be correct with probability $q$, which is the same for everyone. Condorcet showed that the collective procedure which is most likely to yield the best alternative is majority rule: each person reports his/her evidence, and the alternative which gets the most reports is chosen. If we think of democratic elections as a way of divining a group's collective wisdom, then Condorcet's result can be understood as an argument for majority rule voting.

Starting from Condorcet's original model, a recent line of research has considered whether people want to report their evidence truthfully. This is a practical consideration; for example, in Olympic diving competitions, if the US judge always scores US divers highly regardless of their true performance, the Russian judge might respond by always scoring non-US divers highly regardless of their true performance, thereby degrading the quality of the collective decision for everyone. In Olympic diving, the highest and lowest scores of a panel of judges are discarded and only the remaining scores are averaged to obtain a diver's final score, thereby limiting the ability of a single judge to influence the final score. A procedure is called "incentive compatible" for a person if she wants to report her information truthfully given the procedure and given that everyone else is reporting truthfully.

If everyone is identical, strategic considerations do not arise (McLennan, 1998): no individual wants to deviate from a socially optimal procedure because socially optimal is synonymous with individually optimal. The model becomes nontrivial and more realistic when people have different "biases." People might have different prior beliefs over which alternative is best. People might also have different preferences: for example, choosing $b$ when we should have chosen $a$ might be not so bad for you but disastrous for me, and thus I might require a higher "burden of proof" for choosing $b$. Given people's biases, and given a voting procedure such as majority rule or unanimity rule, we can model the situation as a game in which each person chooses what to report given her evidence.

The usual approach in this line of research is to find Nash equilibria of this game, in other words to predict voting behavior given a particular voting procedure. This paper takes the mechanism design approach, considering a large set of possible procedures. I consider anonymous procedures, in which the only thing which affects the group's choice of $a$ or $b$ is the total number of people who report $a$, not who these people are. I also consider only the equilibrium in which everyone reports his/her evidence truthfully, and hence everyone's evidence affects the decision equally. Equality is a well-known argument for anonymous procedures (Riker, 1982). Another argument is in terms of simplicity: since each person's evidence favors either $a$ or $b$, a general procedure must 
consider $2^{n}$ cases, while an anonymous procedure considers only $n+1$ cases, since the total number of people whose evidence favors $a$ ranges from 0 to $n$.

This paper shows that the set of anonymous incentive compatible procedures has two important properties when there are significant biases in both directions (at least one person is significantly biased toward $a$ and at least one person is significantly biased toward $b$ ). First, if an anonymous procedure is incentive compatible when there are significant biases in both directions, then it is incentive compatible for any person, regardless of his preferences and prior beliefs. In other words, it is robust in the sense that it is incentive compatible for all possible biases. Second, if an anonymous procedure is incentive compatible when there are significant biases in both directions, then if it is not trivial, it must be nonmonotonic: an additional vote for $a$ can decrease the probability of the group choosing $a$.

I define one such nonmonotonic procedure, the supermajority penalty (SP) procedure, in which if a weak majority of people report $a$, then the procedure chooses $a$, but if too many people, a supermajority, report $a$, then $b$ is chosen with some probability, thereby "penalizing" the supermajority. When there are significant biases in both directions, I show that the SP procedure is the anonymous incentive compatible procedure which maximizes the utility of an unbiased person, whose priors or preferences do not ex ante favor either alternative. In this sense the SP procedure is optimal and robust: one can approach Condorcet's situation with no knowledge of the priors and preferences of the people involved and be confident in the SP procedure. Finally, I show that as $n$ grows large, the welfare performance of the SP procedure approaches that of majority rule.

\section{THE MODEL}

Condorcet's model has been presented in many papers (here we follow the notation in Chwe, 1999). There is a group of people $N=\{1,2, \ldots, n\}$, where $n$ is odd and at least 3. The group chooses between alternatives $a$ or $b$. Each person receives private evidence on whether $a$ or $b$ is objectively superior. Each person $i \in N$ has a prior belief that $a$ is superior with probability $\pi_{i}(a)$ and $b$ is superior with probability $\pi_{i}(b)$, where $\pi_{i}(a), \pi_{i}(b) \in[0,1]$, and $\pi_{i}(a)+\pi_{i}(b)=1$. Each person's private evidence is correct with probability $q \in(1 / 2,1)$. Let $g(d, e)$ be the probability that a person's private evidence supports $e$ when the superior alternative truly is $d$; thus we have $g(d, e)=q$ if $d=e$ and $g(d, e)=1-q$ if $d \neq e$.

Each person reports her private evidence to the procedure, which outputs the group's decision. The decision procedure is thus a function $f:\{a, b\}^{n} \times\{a, b\} \rightarrow[0,1]$, where $f\left(r_{1}, \ldots, r_{n}, a\right)$ is the probability of choosing $a$ and $f\left(r_{1}, \ldots, r_{n}, b\right)$ is the probability of choosing $b$ given the reports $r_{1}, \ldots, r_{n} \in\{a, b\}$, and of course $f\left(r_{1}, \ldots, r_{n}, a\right)+$ $f\left(r_{1}, \ldots, r_{n}, b\right)=1$. After the decision is made, each person gets utility $u_{i}(d, c)$ when the superior alternative truly is $d$ and the alternative chosen is $c$. We assume that $u_{i}(a, a)>$ $u_{i}(a, b)$ and $u_{i}(b, b)>u_{i}(b, a)$; in other words, everyone prefers the superior alternative.

Each person's strategy is a choice of what to report given his evidence, a function $s_{i}:\{a, b\} \rightarrow\{a, b\}$. The identity function $i d$, where $i d(a)=a$ and $i d(b)=b$, is the 
strategy in which a person always reports his evidence truthfully. Let $s_{a a}$ be the strategy of reporting $a$ all the time $\left(s_{a a}(a)=s_{a a}(b)=a\right)$ and let $s_{b b}$ be the strategy of reporting $b$ all the time $\left(s_{b b}(a)=s_{b b}(b)=b\right)$.

Given the procedure $f$ and strategies $s_{1}, \ldots, s_{n}$, the probability that the group chooses alternative $c$ given that the superior alternative truly is $d$ is $p_{d c}\left(f, s_{1}, \ldots, s_{n}\right)=\sum_{\left(e_{1}, \ldots, e_{n}\right) \in\{a, b\}^{n}} g\left(d, e_{1}\right) \cdots g\left(d, e_{n}\right) f\left(s_{1}\left(e_{1}\right), \ldots, s_{n}\left(e_{n}\right), c\right)$. Here $e_{1}, \ldots, e_{n}$ is the evidence, $s_{1}\left(e_{1}\right), \ldots, s_{n}\left(e_{n}\right)$ are the reports given this evidence, and $f\left(s_{1}\left(e_{1}\right), \ldots, s_{n}\left(e_{n}\right), c\right)$ is the probability of choosing $c$ given the reports. Hence, given strategies $s_{1}, \ldots, s_{n}$, and the procedure $f$, person $i$ 's expected utility is $E U_{i}\left(f, s_{1}, \ldots, s_{n}\right)=\pi_{i}(a) p_{a a}\left(f, s_{1}, \ldots, s_{n}\right) u_{i}(a, a)+\pi_{i}(a) p_{a b}\left(f, s_{1}, \ldots, s_{n}\right) u_{i}(a, b)+$ $\pi_{i}(b) p_{b a}\left(f, s_{1}, \ldots, s_{n}\right) u_{i}(b, a)+\pi_{i}(b) p_{b b}\left(f, s_{1}, \ldots, s_{n}\right) u_{i}(b, b)$.

Because $p_{a b}=1-p_{a a}$ and $p_{b a}=1-p_{b b}$, we have $E U_{i}\left(f, s_{1}, \ldots, s_{n}\right)=$ $\pi_{i}(a) u_{i}(a, b)+\pi_{i}(b) u_{i}(b, a)+\pi_{i}(a)\left(u_{i}(a, a)-u_{i}(a, b)\right) p_{a a}\left(f, s_{1}, \ldots, s_{n}\right)+\pi_{i}(b)\left(u_{i}(b, b)-\right.$ $\left.u_{i}(b, a)\right) p_{b b}\left(f, s_{1}, \ldots, s_{n}\right)$. The first two terms here are constants and can be dropped. We can then normalize and write

$$
E U_{i}\left(f, s_{1}, \ldots, s_{n}\right)=\phi_{i}(a) p_{a a}\left(f, s_{1}, \ldots, s_{n}\right)+\phi_{i}(b) p_{b b}\left(f, s_{1}, \ldots, s_{n}\right),
$$

where $\phi_{i}(a), \phi_{i}(b)$ are defined by

$$
\begin{aligned}
\phi_{i}(a) & =\frac{\pi_{i}(a)\left(u_{i}(a, a)-u_{i}(a, b)\right)}{\pi_{i}(a)\left(u_{i}(a, a)-u_{i}(a, b)\right)+\pi_{i}(b)\left(u_{i}(b, b)-u_{i}(b, a)\right)} \\
\phi_{i}(b) & =\frac{\pi_{i}(b)\left(u_{i}(b, b)-u_{i}(b, a)\right)}{\pi_{i}(a)\left(u_{i}(a, a)-u_{i}(a, b)\right)+\pi_{i}(b)\left(u_{i}(b, b)-u_{i}(b, a)\right)} .
\end{aligned}
$$

Note that $\phi_{i}(a), \phi_{i}(b) \in[0,1]$ and $\phi_{i}(a)+\phi_{i}(b)=1$.

The parameters $\phi_{i}(a), \phi_{i}(b)$ represent the "bias" of person $i$. If everyone has the same prior belief $\pi_{i}(a)=\pi_{i}(b)=1 / 2$, then $\phi_{i}(a), \phi_{i}(b)$ correspond to the relative magnitudes of $u_{i}(a, a)-u_{i}(a, b)$ and $u_{i}(b, b)-u_{i}(b, a)$. For example, if $\pi_{i}(a)=\pi_{i}(b)=1 / 2$ and $u_{i}(a, a)=2, u_{i}(a, b)=0, u_{i}(b, a)=0, u_{i}(b, b)=1$, then $\phi_{i}(a)=2 / 3$ and $\phi_{i}(b)=1 / 3$; person $i$ is biased toward $a$ because her payoff from choosing $a$ correctly is twice that of choosing $b$ correctly. If one has the "standard" utility function $u_{i}(a, a)=1, u_{i}(a, b)=$ $0, u_{i}(b, a)=0, u_{i}(b, b)=1$, then $\phi_{i}(a)=\pi_{i}(a)$ and $\phi_{i}(b)=\pi_{i}(b)$; the bias $\phi_{i}$ is simply the prior belief $\pi_{i}$. If $\phi_{i}(a)>\phi_{i}(b)$, either because of $u_{i}$ or $\pi_{i}$, then we say that person $i$ is "biased toward" $a$. If a person has bias $\phi_{i}(a)=\phi_{i}(b)=1 / 2$, we call that person unbiased.

The sum of everyone's utility is simply

$\sum_{i \in N} E U_{i}\left(f, s_{1}, \ldots, s_{n}\right)=\left[\sum_{i \in N} \phi_{i}(a)\right] p_{a a}\left(f, s_{1}, \ldots, s_{n}\right)+\left[\sum_{i \in N} \phi_{i}(b)\right] p_{b b}\left(f, s_{1}, \ldots, s_{n}\right)$.

Thus utility averaged over the group is equal to the utility of a person who has an average bias. If average bias in a group is $\phi_{i}(a)=\phi_{i}(b)=1 / 2$, then maximizing the sum of everyone's utility is the same as maximizing the utility of an unbiased person. 
We say that the procedure $f$ is incentive compatible for person $i$ if the following two incentive compatibility (IC) constraints hold:

$$
\begin{aligned}
& E U_{i}(f, i d, \ldots, i d) \geq E U_{i}\left(f, i d, \ldots, s_{a a}, \ldots, i d\right) \\
& E U_{i}(f, i d, \ldots, i d) \geq E U_{i}\left(f, i d, \ldots, s_{b b}, \ldots, i d\right) .
\end{aligned}
$$

In other words, reporting truthfully is at least as good as reporting $a$ always or reporting $b$ always, given that everyone else reports truthfully. We do not have to consider the "always lie" strategy $s_{b a}$, defined by $s_{b a}(a)=b$ and $s_{b a}(b)=a$, because if one does not gain by sometimes misreporting, then one does not gain by always misreporting. We say the procedure $f$ is incentive compatible if it is incentive compatible for all $i \in N$; in other words, $(i d, \ldots, i d)$ is a Nash equilibrium.

For $r \in\{a, b\}^{n}$, define $\alpha(r)=\#\left\{i \in N: r_{i}=a\right\}$; in other words, given the vector of reports $r, \alpha(r)$ is the number of people who report $a$. We say that the procedure $f$ is anonymous if $\alpha(r)=\alpha\left(r^{\prime}\right) \Rightarrow f(r, a)=f\left(r^{\prime}, a\right)$. In other words, an anonymous procedure depends only on the number of people who report $a$ or $b$, not their identities. An anonymous procedure $f$ can be represented by the numbers $\gamma(0), \gamma(1), \ldots \gamma(n)$, where $\gamma(\alpha(r))=f(r, a)$; in other words, $\gamma(j)$ is the probability that $a$ is chosen given that there are $j$ reports of $a$.

We say that a procedure $f$ is symmetric if $f(r, a)=f\left(r^{\prime}, b\right)$ for all $r, r^{\prime}$ such that $r_{i}=a \Leftrightarrow r_{i}^{\prime}=b$. In other words, if $r, r^{\prime}$ are exact "opposites" in that each person's report in $r$ is the opposite of her report in $r^{\prime}$, then the probability of choosing $a$ given $r$ is the same as the probability of choosing $b$ given $r^{\prime}$. If a procedure $f$ is both anonymous and symmetric, and the probability of choosing $a$ given $j$ reports of $a$ is $\gamma(j)$, then we have $\gamma(j)=1-\gamma(n-j)$; the probability of choosing $a$ given $j$ reports of $a$ is equal to the probability of choosing $b$ given $j$ reports of $b$. We say that a procedure $f$ is trivial if there exists a constant $\kappa \in[0,1]$ such that $f(r, a)=\kappa$ for all $r \in\{a, b\}^{n}$. A trivial procedure chooses $a$ with the same probability regardless of the reports. We say that a procedure $f$ is monotonic if $\left\{j \in N: r_{j}=a\right\} \subset\left\{j \in N: r_{j}^{\prime}=a\right\} \Rightarrow f(r, a) \leq f\left(r^{\prime}, a\right)$. In other words, if the set of people who report $a$ increases, then the probability of choosing $a$ does not decrease. An anonymous procedure is monotonic if $\gamma(0) \leq \gamma(1) \leq \cdots \leq \gamma(n)$.

\section{RESULTS}

Our main results are best understood as resulting from Lemma 1: when a procedure is anonymous and symmetric, a person's bias can be "factored out" of her incentive compatibility constraints (all proofs are in the Appendix).

Lemma 1 Say that $f$ is anonymous and symmetric. Then person i's two incentive compatibility constraints can be written as:

$$
\begin{aligned}
& \left(\phi_{i}(a)-q\right) W(q, \gamma) \geq 0 \\
& \left(\phi_{i}(b)-q\right) W(q, \gamma) \geq 0,
\end{aligned}
$$


where $\gamma(\alpha(r))=f(r, a)$ and $W(q, \gamma)=\sum_{j=0}^{n-1} C_{j}^{n-1} q^{j}(1-q)^{n-1-j}(\gamma(j)-\gamma(j+1))$, and $C_{j}^{n}$ is the binomial coefficient $C_{j}^{n}=n ! /((n-j) ! j !)$.

In other words, when a procedure is anonymous and symmetric, the incentive compatibility constraints boil down to the sign of $W(q, \gamma)$. When a person is relatively unbiased, with $\phi_{i}(a) \leq q$ and $\phi_{i}(b) \leq q$, then $\phi_{i}(a)-q$ and $\phi_{i}(b)-q$ and are both nonpositive (at least one is negative), and thus we have $W(q, \gamma) \leq 0$. When a person is significantly biased, with $\phi_{i}(a)>q$ or $\phi_{i}(b)>q$, then $\phi_{i}(a)-q$ and $\phi_{i}(b)-q$ have different signs and we have $W(q, \gamma)=0$.

Here is an intuitive explanation of Lemma 1, which is basically a restatement of the proof. Remember that a person's bias toward $a$ can be understood as a prior belief $\phi(a)$ that $a$ is truly superior. Say a person has evidence $b$ and is considering whether to report $a$. She must consider two events. The first is the event the superior alternative is $a$; since her evidence is $b$, the probability of this event is $\phi(a)(1-q)$. In this event, it might be good for her to lie and report $a$ instead of $b$, because this might increase the probability of choosing the truly superior $a$. How much this probability changes depends on the procedure and what everyone else's evidence is (and also what everyone else's strategy is, but we assume that everyone else tells the truth). Call this probability difference $\Delta_{a}$. The second is the event that the superior alternative is $b$; since her evidence is $b$, the probability of this state of the world is $\phi(b) q$. In this event, by lying the person foregoes the benefit of telling the truth. Telling the truth and reporting $b$ might yield a higher probability of choosing the truly superior $b$, relative to lying and reporting $a$. Call this probability difference $\Delta_{b}$. For the procedure to be incentive compatible, the benefit gained from lying must be less than the benefit foregone, the benefit from reporting the truth. In other words, $\phi(a)(1-q) \Delta_{a} \leq \phi(b) q \Delta_{b}$.

Remember that $\Delta_{a}$ is conditional on the event that $a$ is truly superior and the person receives evidence for $a$, and $\Delta_{b}$ is conditional on the event that $b$ is truly superior and the person receives evidence for $a$. Remember that $\Delta_{a}$ is the difference in probability of choosing $a$ if the person reports $a$ as opposed to $b$ and $\Delta_{b}$ is the difference in probability of choosing $b$ if the person reports $b$ as opposed to $a$. When the procedure is symmetric, it is easy to see that $\Delta_{a}=\Delta_{b}$; these (conditional) probability differences are equal. Thus we have $(\phi(a)(1-q)-\phi(b) q) \Delta_{a} \leq 0$, and hence $(\phi(a)-q \phi(a)-q+q \phi(a)) \Delta_{a} \leq 0$, which is the first inequality in Lemma 1 , when we define $W(q, \gamma)=-\Delta_{a}$. Showing $\left(\phi_{i}(b)-q\right) W(q, \gamma) \geq 0$, the second inequality in Lemma 1 , is similar.

Now that we have Lemma 1, our results follow quickly. Our first proposition is that if an anonymous procedure is incentive compatible for a person who is significantly biased toward $a$ and also for a person who is significantly biased toward $b$, then the incentive compatibility constraints hold with equality for any person, regardless of her bias.

Proposition 1 Say that there exist $i, j \in N$ such that $\phi_{i}(b)>q$ and $\phi_{j}(a)>q$. Iff is anonymous and incentive compatible for persons $i$ and $j$, then person l's incentive compatibility constraints hold with equality for any $\phi_{l}$. 
The proof of Proposition 1 is immediate when $f$ is symmetric. When $\phi_{i}(b)>q$, by Lemma 1 we have $W(q, \gamma)=0$, and thus $f$ is incentive compatible for any bias $\phi_{l}$. When $f$ is not symmetric, we use the additional fact that $\phi_{j}(a)>q$.

Proposition 2 says that when there are significant biases in both directions, a nontrivial anonymous incentive compatible procedure cannot be monotonic.

Proposition 2 Say that there exist $i, j \in N$ such that $\phi_{i}(b)>q$ and $\phi_{j}(a)>q$. Say $f$ is an anonymous incentive compatible procedure which is not trivial. Then $f$ is not monotonic.

In other words, in any nontrivial anonymous incentive compatible procedure, there is at least one scenario in which an additional report for $a$ makes the probability that $a$ is chosen strictly decrease. Again, the proof is immediate when $f$ is symmetric. From Lemma 1 , we have $W(q, \gamma)=0$. But $W(q, \gamma)$ is a weighted sum of $\gamma(j)-\gamma(j+1)$ terms, where all the weights are positive. So either $\gamma(j)-\gamma(j+1)=0$ for all $j$, in which case $f$ is trivial, or $\gamma(j)-\gamma(j+1)>0$ for some $j$, in which case $f$ is not monotonic.

Now we know that when there are significant biases in both directions, any anonymous incentive compatible procedure is nonmonotonic and robust in the sense that it is incentive compatible for all biases. Among all anonymous incentive compatible procedures, which might be the best? I define the SP procedure $f_{S P}$ and show that it maximizes the expected utility of an unbiased person. Given $q$ and $n$, Lemma 2 provides two numbers $k$ and $z$, which are parameters of $f_{S P}$.

Lemma 2 Given $q$ and $n$, there uniquely exists $k \in\{0,1, \ldots,(n-3) / 2\}$ such that

$$
z=\frac{D_{k y^{(n-1) / 2}}-k\left(y^{k-1}+y^{n-k}\right)}{(n-k)\left(y^{k}+y^{n-1-k}\right)-k\left(y^{k-1}+y^{n-k}\right)} \in(0,1],
$$

where $y=q /(1-q)$ and $D_{k}=(k !(n-k) !) /(((n-1) / 2) !)^{2}$. Also, $k$ is nonincreasing in $q$ and $k<(1-q) n$. As $n$ groms large, $k / n \rightarrow \rho$, where $\rho<1-q$ is uniquely defined by $\rho \log \rho+(1-\rho) \log (1-\rho)+\log 2=(1 / 2-\rho) \log y$.

Definition. Given $q$ and $n$, define the SP procedure $f_{S P}$ as

$$
f_{S P}(r, a)= \begin{cases}1 & \text { if } \alpha(r)<k \\ z & \text { if } \alpha(r)=k \\ 0 & \text { if } k<\alpha(r) \leq(n-1) / 2 \\ 1 & \text { if }(n+1) / 2 \leq \alpha(r)<n-k \\ 1-z & \text { if } \alpha(r)=n-k \\ 0 & \text { if } \alpha(r)>n-k\end{cases}
$$

where $k \in\{0,1, \ldots,(n-3) / 2\}$ and $z \in(0,1]$ are defined as in Lemma 2 , and $f_{S P}(r, b)=$ $1-f_{S P}(r, a)$.

In other words, if a weak majority of the reports are for $a$, then $a$ is chosen with probability 1 . However, if the number of reports for $a$ is greater than $n-k$, then this 


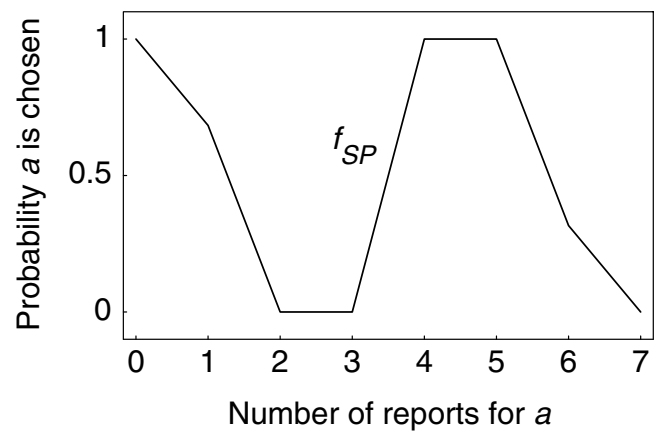

Figure 1. $f_{S P}$ when $n=7$ and $q=2 / 3$ (thus $k=1$ and $z=95 / 139$ ).

supermajority is "penalized" and $a$ is chosen with probability 0 . If the number of reports for $a$ is equal to $n-k$, then $a$ is chosen with probability $1-z$. For example, when $n=7$ and $q=2 / 3$, Lemma 2 gives us $k=1$ and $z=95 / 139$, and the SP procedure $f_{S P}$ is shown in Figure 1.

Proposition 3 Say that there exist $i, j \in N$ such that $\phi_{i}(b)>q$ and $\phi_{j}(a)>q$. Then $f_{S P}$ is the anonymous incentive compatible procedure which uniquely maximizes $E U_{0}$, where $\phi_{0}(a)=\phi_{0}(b)=1 / 2$.

If $\phi_{i}(a) \in[1-q, q]$ for all $i \in N$, then the optimal anonymous incentive compatible procedure, from the point of view of an unbiased person, is majority rule. But if a person's bias is outside $[1-q, q]$, then majority rule is no longer incentive compatible. If some people have $\phi_{i}(a)$ slightly greater than $q$ and everyone else has $\phi_{j}(a) \in[1-q, q]$, then the optimal anonymous incentive compatible procedure allows $a$ to be chosen with some probability when it gets one report short of a majority (Chwe, 1999). If there is at least one person with $\phi_{i}(a)<1-q$ and at least one with $\phi_{j}(a)>q$, then Proposition 3 applies and the unique optimal anonymous incentive compatible procedure is the SP procedure.

How often is the supermajority penalized? In expectation we either have $q n$ reports for $a$ (if $a$ is the objectively superior alternative) or $(1-q) n$ reports for $a$ (if $b$ is the objectively superior alternative). Lemma 2 says that $k<(1-q) n$, and hence the expected number of reports is not in the supermajority penalty region. Lemma 2 also says that as $n$ grows large, $k / n \rightarrow \rho$, where $\rho<1-q$ is a constant which does not depend on $n$. Thus as $n$ grows large, the law of large numbers applies and the supermajority penalty regions are almost never reached. We thus state Proposition 4 without proof.

Proposition 4 As $n$ grows large, $E U_{i}\left(f_{S P}, i d, \ldots, i d\right)$ approaches $E U_{i}\left(f_{M R}, i d, \ldots, i d\right)$, where $f_{M R}$ is majority rule, defined as $f_{M R}(r, a)=1$ if $\alpha(r) \geq(n+1) / 2$ and $f_{M R}(r, a)=0$ otherwise.

Figure 2 shows how the SP procedure changes when $q=2 / 3$ and $n$ goes from 5 to 101. When $n=101$ for example, given that $q=2 / 3$, the number of reports for $a$ cluster 

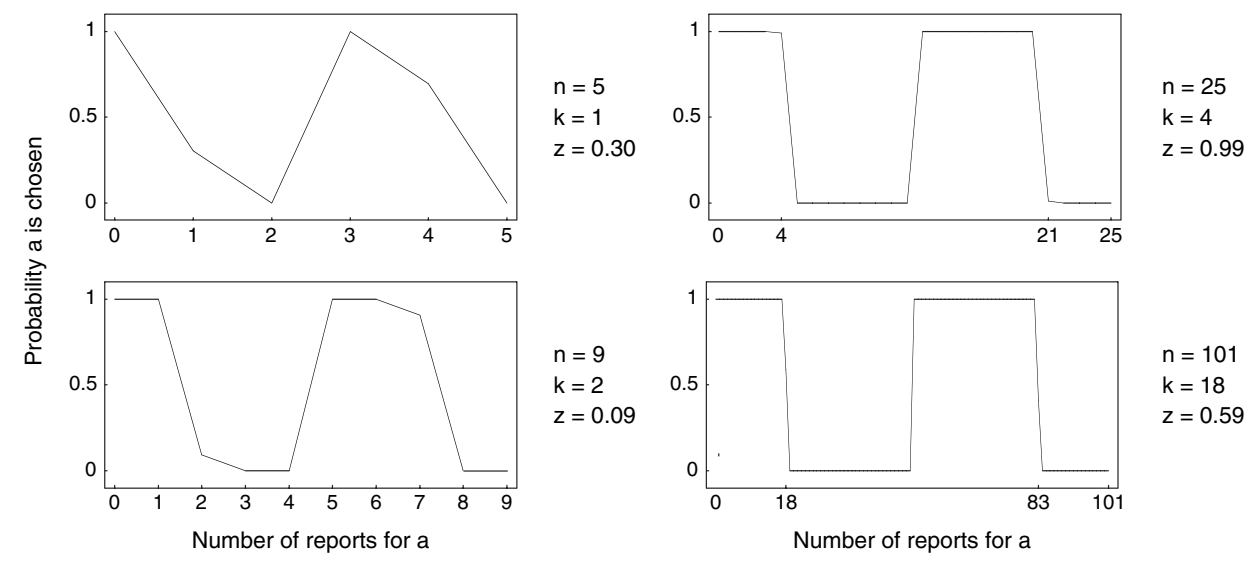

Figure 2. $f_{S P}$ when $q=2 / 3$ and $n=5,9,25,101$.
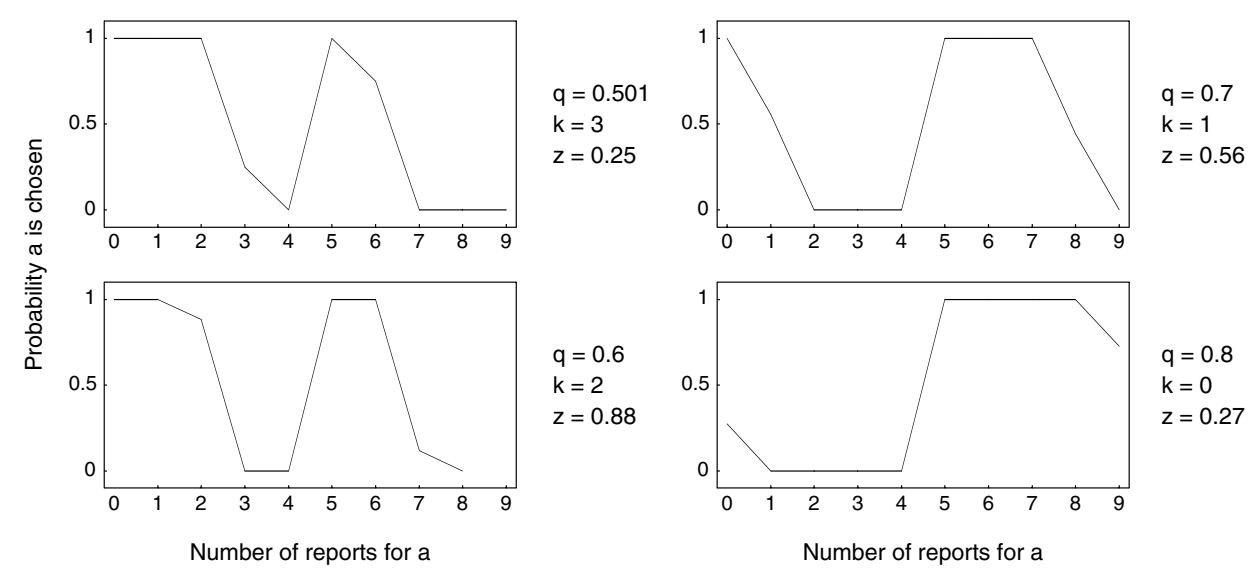

Figure 3. $f_{S P}$ when $n=9$ and $q=0.501,0.6,0.7,0.8$.

around 34 (if $b$ is superior) or 67 (if $a$ is superior) and the supermajority penalty rarely occurs.

As $n$ grows large, the SP procedure's welfare performance approaches that of majority rule, the "first best" procedure if there are no incentive compatibility constraints. It does so while eliciting truthful reports from everyone, regardless of their bias. When $n$ is large, the supermajority penalty regions are enough to discourage lying even though they are rarely reached. The reason for this is as $n$ grows large, the temptation to lie also becomes small, since lying "helps" only when everyone else's reports are equally divided, an event which also becomes rare.

How does the SP procedure depend on the quality of evidence $q$ ? Lemma 2 says that $k$ is nonincreasing in $q$ : as $q$ increases, $k$ stays the same or decreases. Figure 3 shows this 
for $n=9$ and $q$ going from 0.501 to 0.8 . As $q$ increases, each person's evidence gets stronger and people's biases become relatively less important; thus the "distortion" in the procedure necessary to deal with the biases lessens, and the supermajority penalty regions shrink. As $q$ approaches 1 , we know $k=0$ because Lemma 2 says that $k<(1-q) n$, and it is also easy to see from the definition of $z$ in Lemma 2 that as $q$ approaches 1 , $z$ approaches 0 . Hence as $q$ approaches 1 , the SP procedure approaches majority rule.

\section{EXAMPLE}

An example is helpful in explaining the results and how they are proved. Say that $n=3$ and thus an anonymous procedure $\gamma$ can be written as four numbers $\gamma(0), \gamma(1), \gamma(2), \gamma(3)$, where $\gamma(j) \in[0,1]$ is the probability that the group chooses $a$ given $j$ reports for $a$.

Remember that a person's expected utility is given by the formula $(*)$ earlier. The first incentive compatibility constraint is that whenever a person receives evidence for $b$, she should report $b$ and not $a$. Given that she receives evidence for $b$, her expected utility from reporting $b$ is

$$
\begin{aligned}
& \phi(a)(1-q)\left[q^{2} \gamma(2)+2 q(1-q) \gamma(1)+(1-q)^{2} \gamma(0)\right] \\
& \quad+\phi(b) q\left[(1-q)^{2}(1-\gamma(2))+2(1-q) q(1-\gamma(1))+q^{2}(1-\gamma(0))\right] .
\end{aligned}
$$

The first line is her bias $\phi(a)$ times the probability that $a$ is chosen when $a$ is truly the superior alternative. When $a$ is truly the superior alternative, the probability that she gets evidence $b$ is $1-q$, and we have three cases. In the first case, two other people get evidence $a$, which happens with probability $q^{2}$, and the procedure chooses $a$ with probability $\gamma(2)$, and so forth. The second line is her bias $\phi(b)$ times the probability that $b$ is chosen when $b$ truly is the superior alternative. When $b$ is truly the superior alternative, the probability that she gets evidence $b$ is $q$, and we similarly have three cases.

If when she receives evidence for $b$ she reports $a$ instead, her expected utility becomes

$$
\begin{aligned}
& \phi(a)(1-q)\left[q^{2} \gamma(3)+2 q(1-q) \gamma(2)+(1-q)^{2} \gamma(1)\right] \\
& \quad+\phi(b) q\left[(1-q)^{2}(1-\gamma(3))+2(1-q) q(1-\gamma(2))+q^{2}(1-\gamma(1))\right],
\end{aligned}
$$

which is the same as before except that all the arguments of $\gamma$ increase by 1 . By reporting $a$ instead of $b$, she increases the number of $a$ reports by 1 .

So the incentive compatibility constraint is simply that the first expression minus the second expression is greater than or equal to zero:

$$
\begin{aligned}
& \phi(a)(1-q)\left[q^{2}(\gamma(2)-\gamma(3))+2 q(1-q)(\gamma(1)-\gamma(2))+(1-q)^{2}(\gamma(0)-\gamma(1))\right] \\
& \quad+\phi(b) q\left[(1-q)^{2}(\gamma(3)-\gamma(2))+2(1-q) q(\gamma(2)-\gamma(1))+q^{2}(\gamma(1)-\gamma(0))\right] \geq 0
\end{aligned}
$$

or in other words

$$
\begin{aligned}
& \phi(a)(1-q)\left[q^{2}(\gamma(2)-\gamma(3))+2 q(1-q)(\gamma(1)-\gamma(2))+(1-q)^{2}(\gamma(0)-\gamma(1))\right] \\
& \quad-\phi(b) q\left[(1-q)^{2}(\gamma(2)-\gamma(3))+2(1-q) q(\gamma(1)-\gamma(2))+q^{2}(\gamma(0)-\gamma(1))\right] \geq 0 .
\end{aligned}
$$


Assume for a moment that $f$ is symmetric (this is justified later). Then we have $\gamma(3)=1-\gamma(0)$ and $\gamma(2)=1-\gamma(1)$ and thus $\gamma(2)-\gamma(3)=\gamma(0)-\gamma(1)$. Thus we can write this inequality as:

$$
(\phi(a)(1-q)-\phi(b) q) W(q, \gamma) \geq 0,
$$

where $W(q, \gamma)=(1-q)^{2}(\gamma(0)-\gamma(1))+2(1-q) q(\gamma(1)-\gamma(2))+q^{2}(\gamma(2)-\gamma(3))$. Since $\phi(b)=1-\phi(a)$, we have $\phi(a)(1-q)-\phi(b) q=\phi(a)-q$, and we $\operatorname{get}(\phi(a)-q) W(q, \gamma) \geq 0$. Similarly, the second incentive compatibility constraint, that whenever a person receives evidence for $a$ she should report $a$ and not $b$, turns out to be $(\phi(b)-q) W(q, \gamma) \geq 0$.

This is the proof of Lemma 1 when $n=3$. As mentioned before, when a person is not very biased and $\phi(a), \phi(b) \in[1-q, q]$, the two constraints boil down to $W(q, \gamma) \leq 0$. Note that this is satisfied by majority rule, in which $\gamma(0)=\gamma(1)=0$ and $\gamma(2)=\gamma(3)=1$.

As mentioned before, when $\phi(a)>q$ or $\phi(b)>q$, then $\phi(a)-q$ and $\phi(b)-q$ have different signs, and thus $W(q, \gamma)=0$. Thus the procedure is incentive compatible, and the incentive compatibility constraints hold with equality, for any $\phi$ (Proposition 1). Since $W(q, \gamma)=0$, from the definition of $W(q, \gamma)$, either all three terms $\gamma(0)-\gamma(1)$, $\gamma(1)-\gamma(2)$, and $\gamma(2)-\gamma(3)$ are zero, in which case the procedure is trivial, or at least one of the terms is negative, in which case the procedure is nonmonotonic (Proposition 2).

To illustrate the proof of Proposition 3, we write down the expected utility of an unbiased person, a person with bias $\phi(a)=\phi(b)=1 / 2$ :

$$
\begin{aligned}
& \frac{1}{2}\left[q^{3} \gamma(3)+3 q^{2}(1-q) \gamma(2)+3 q(1-q)^{2} \gamma(1)+(1-q)^{3} \gamma(0)\right] \\
& \quad+\frac{1}{2}\left[q^{3}(1-\gamma(0))+3 q^{2}(1-q)(1-\gamma(1))+3 q(1-q)^{2}(1-\gamma(2))\right. \\
& \left.\quad+(1-q)^{3}(1-\gamma(3))\right] .
\end{aligned}
$$

To find the $\gamma$ which maximizes this, all terms which are constant in $\gamma$ can be ignored and we thus have the objective

$$
\begin{aligned}
& \frac{1}{2}\left[\left(q^{3}-(1-q)^{3}\right) \gamma(3)+\left(3 q^{2}(1-q)-3 q(1-q)^{2}\right) \gamma(2)\right. \\
& \left.\quad+\left(3 q(1-q)^{2}-3 q^{2}(1-q)\right) \gamma(1)+\left((1-q)^{3}-q^{3}\right) \gamma(0)\right] .
\end{aligned}
$$

Assuming that the procedure is symmetric, $\gamma(3)=1-\gamma(0)$ and $\gamma(2)=1-\gamma(1)$, and again dropping terms constant in $\gamma$, our objective becomes

$$
\left((1-q)^{3}-q^{3}\right) \gamma(0)+\left(3 q(1-q)^{2}-3 q^{2}(1-q)\right) \gamma(1) .
$$

When one person has $\phi(a)>q$ and another has $\phi(b)>q$, then the incentive compatibility constraint is $W(q, \gamma)=0$, and given that the procedure is symmetric, this simplifies to

$$
\left((1-q)^{2}+q^{2}\right) \gamma(0)+\left(4 q(1-q)-q^{2}-(1-q)^{2}\right) \gamma(1)=2(1-q) q .
$$


Say for example that $q=2 / 3$. Then to find the symmetric anonymous procedure which maximizes the expected utility of an unbiased person, subject to the constraint that the procedure must be incentive compatible, we solve the following constrained maximization problem:

$$
\underset{\gamma(0), \gamma(1) \in[0,1]}{\operatorname{Maximize}}-\frac{7}{27} \gamma(0)-\frac{2}{9} \gamma(1) \text { such that } \frac{5}{9} \gamma(0)+\frac{1}{3} \gamma(1)=\frac{4}{9} .
$$

To maximize the objective, we would like to set $\gamma(0)=\gamma(1)=0$ (majority rule), but this would not satisfy the constraint. At least one of the variables $\gamma(0), \gamma(1)$ must be made positive. Which one? It depends on how much a variable hurts the objective relative to how much it helps satisfy the constraint. If we look at the ratios of the coefficients on $\gamma(0)$ and $\gamma(1)$, we find that the ratio for $\gamma(0)$ is $\left(-\frac{7}{27}\right) /\left(\frac{5}{9}\right)=-\frac{7}{15}$ and the ratio for $\gamma(1)$ is $\left(-\frac{2}{9}\right) /\left(\frac{1}{3}\right)=-\frac{2}{3}$. Since the ratio for $\gamma(0)$ is less negative, making $\gamma(0)$ positive is the better "deal," and the maximum is obtained at $\gamma(0)=4 / 5$ and $\gamma(1)=0$. For general $q$, we show that $\left((1-q)^{3}-q^{3}\right) /\left((1-q)^{2}+q^{2}\right)$ is greater than $\left(3 q(1-q)^{2}-3 q^{2}(1-q)\right) /\left(4 q(1-q)-q^{2}-(1-q)^{2}\right)$.

The only thing remaining is to justify the assumption that $\gamma$ is symmetric. The argument, roughly speaking, proceeds like this. Say for example that we have $q=2 / 3$ and we have biases $\phi_{1}(a)=1 / 4, \phi_{2}(a)=4 / 7, \phi_{3}(a)=10 / 11$; person 1 is biased toward $b$, person 2 is relatively unbiased, and person 3 is biased toward $a$. Note that the IC constraints are linear in $\phi(a)$. Hence if $\gamma$ satisfies the IC constraints for $\phi(a)=1 / 4$ and for $\phi(a)=10 / 11$, then they satisfy the IC constraints for all convex combinations of $1 / 4$ and $10 / 11$, and in particular, $\phi(a)=3 / 4 \in[1 / 4,10 / 11]$. Thus any incentive compatible $\gamma$ satisfies the IC constraints for $\phi(a)=1 / 4$ and $\phi(a)=3 / 4$.

Define a new procedure $\gamma^{\prime \prime}$ as $\gamma^{\prime \prime}(j)=1-\gamma(3-j)$. In other words, the probability that $\gamma^{\prime \prime}$ chooses $a$ given 2 reports of $a$ is the same as the probability that $\gamma$ chooses $b$ given 2 reports of $b$. It is not difficult to show, by simply exchanging the names of $a$ and $b$, that if $\gamma$ satisfies the IC constraints for $\phi(a)=r$, then $\gamma^{\prime \prime}$ satisfies the IC constraints for $\phi(a)=1-r$. Since $\gamma$ satisfies the IC constraints for $\phi(a)=1 / 4$ and $\phi(a)=3 / 4$, we know that $\gamma^{\prime \prime}$ satisfies the IC constraints for $\phi(a)=3 / 4$ and $\phi(a)=1 / 4$. So both $\gamma$ and $\gamma^{\prime \prime}$ satisfy the IC constraints for $\phi(a)=1 / 4$ and $\phi(a)=3 / 4$. Since the IC constraints are linear in $\gamma$, if we define another procedure $\gamma^{\prime}$ as $\gamma^{\prime}(j)=\left(\gamma(j)+\gamma^{\prime \prime}(j)\right) / 2$, we know that $\gamma^{\prime}$ satisfies the IC constraints for $\phi(a)=1 / 4$ and $\phi(a)=3 / 4$.

Note that the procedure $\gamma^{\prime}$ is symmetric. We can think of $\gamma^{\prime}$ as a "symmetrized" version of $\gamma$. Since $\gamma^{\prime}$ is symmetric and satisfies the IC constraints for $\phi(a)=3 / 4>2 / 3=q$, by Lemma $1, \gamma^{\prime}$ satisfies the IC constraints for all $\phi(a)$ and hence is incentive compatible. So any incentive compatible $\gamma$ has a symmetric version $\gamma^{\prime}$ which is incentive compatible.

Remember that our objective function is the expected utility of an unbiased person, with $\phi(a)=1 / 2$. It is easy to show that an unbiased person gets the same expected utility from $\gamma$ and $\gamma^{\prime \prime}$, and thus by convexity gets the same expected utility from $\gamma^{\prime}$. We can thus safely restrict ourselves to symmetric $\gamma$, because for any incentive compatible $\gamma$ which is not symmetric, there is a symmetric version $\gamma^{\prime}$ which yields the same expected utility. 
To summarize, what makes things simple is that for anonymous and symmetric procedures, the terms $\phi(a)-q$ and $\phi(b)-q$ factor out of the IC constraints (Lemma 1), and thus incentive compatibility boils down to the sign of $W(q, \gamma)$. To understand intuitively why the SP procedure has the shape that it does in general, one simply looks at the ratio of the coefficient of $\gamma(j)$ in the objective function $E U_{0}$ to the coefficient of $\gamma(j)$ in the constraint $W(q, \gamma)=0$. This ratio always decreases (becomes more negative) in $j$. Making $\gamma(0)$ positive is always the best "deal," and when this is maxed out at $\gamma(0)=1$, we resort to the next best deal, $\gamma(1)$, until this is maxed out, and so forth.

\section{DISCUSSION}

This paper looks only at anonymous procedures and only the equilibrium in which everyone tells the truth. Also, the welfare criterion in Proposition 3 is the utility of an exactly unbiased person. I discuss these two assumptions in turn.

We assume that everyone's evidence matters equally in the decision and thus we consider anonymous procedures. But even if the procedure is anonymous, if a person for example always reports $a$ regardless of her evidence, then her evidence does not affect the decision and is effectively discarded. Thus to make sure that everyone's evidence matters equally, we also assume the equilibrium in which everyone reports truthfully. But other Nash equilibria are possible. Say $n=2 / 3$ and $q=2 / 3$. As shown in our example earlier, the SP procedure is given by $\gamma(0)=4 / 5, \gamma(1)=0, \gamma(2)=1, \gamma(3)=1 / 5$. Say $\phi_{1}(a)=3 / 4, \phi_{2}(a)=1 / 4$, and $\phi_{3}(a)=1 / 2$. In other words, person 1 is biased toward $a$, person 2 is biased toward $b$, and person 3 is unbiased. By Proposition 3 we know that $(i d, i d, i d)$ is a Nash equilibrium, which gives everyone expected utility $8 / 15 \approx 0.533$. However, it turns out that $\left(s_{a a}, s_{b b}, i d\right)$ is also a Nash equilibrium, which gives everyone expected utility $2 / 3 \approx 0.667$. Here person 1 always reports $a$ and person 2 always reports $b$, and thus their evidence does not affect the decision; this is equivalent to a procedure in which only person 3's vote is counted. In this case, it seems odd to assume that everyone tells the truth when everyone would prefer another equilibrium.

There are two ways to approach this issue. The first is to evaluate a procedure by finding all equilibria given the procedure. But even for the simplest procedures such as majority rule, there are many equilibria. For example, if $n=3$ and everyone is unbiased, given majority rule, the set of Nash equilibria includes $(i d, i d, i d),\left(s_{a a}, s_{b b}, i d\right)$, $\left(s_{a a}, s_{a a}, s_{a a}\right)$, and $\left(s_{b b}, s_{b b}, s_{b b}\right)$; to make the seemingly obvious argument that majority rule is optimal when everyone is unbiased, we also have to assume outright the truthful equilibrium.

Short of finding all equilibria for a given procedure, we can look at cases in which one equilibrium seems particularly justifiable. For example, say that $m$ people are " $a$ partisans" who have bias $\phi(a)=1$ and $m$ people are " $b$-partisans" who have bias $\phi(a)=0$, and everyone else is an "independent," with $\phi(a)=1 / 2$ (as in for example Feddersen and Pesendorfer, 1996). An $a$-partisan wants the group to choose $a$, and a $b$-partisan wants the group to choose $b$, regardless of the evidence. Under majority rule, a weakly dominant strategy for an $a$-partisan is to report $a$ all the time, and a weakly dominant strategy for 


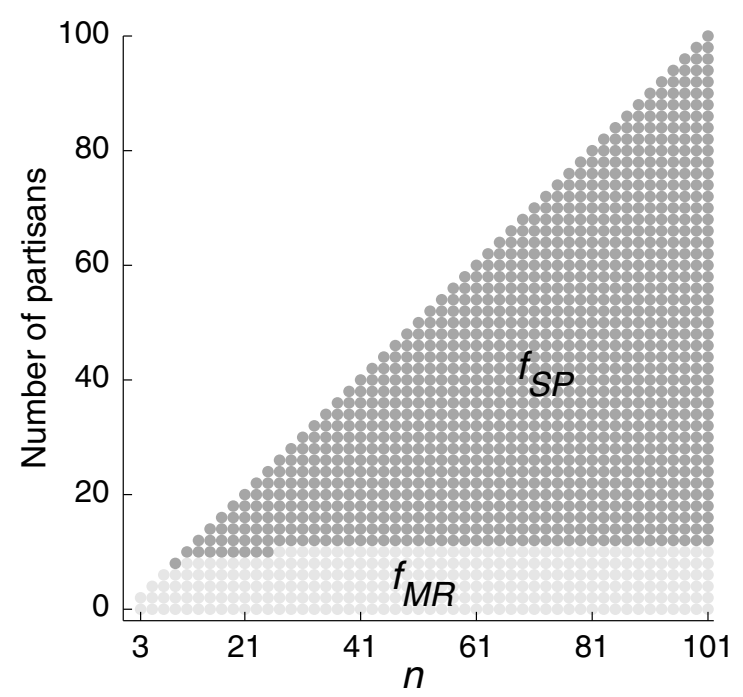

Figure 4 . Which procedure, $f_{S P}$ or $f_{M R}$, gives a higher $E U_{0}$ when partisans have bias $\phi(a)=1$ or $\phi(a)=0$ and everyone else has $\phi(a)=1 / 2$, where $q=2 / 3$.

a $b$-partisan is to report $b$ all the time. If the independents all report truthfully, then this is an equilibrium, in which the partisans cancel each other out and the independents effectively make the decision. We compare majority rule assuming this equilibrium with the SP procedure assuming the equilibrium in which everyone always reports truthfully. Figure 4 shows which one is better at maximizing the expected utility of an unbiased person, depending on the number of partisans, where $n$ ranges from 3 to 101 and $q=2 / 3$.

For $n=3,5,7$, majority rule is always superior, even if almost everyone is a partisan (there is always at least one independent). For example, when $n=7$, even if three people are $a$-partisans and three are $b$-partisans, leaving the group to depend only on the information of the single independent, majority rule is better than using the SP procedure. However, when $n=9$ and there are eight partisans (four on each side), then the SP procedure is better than relying on the single independent. The SP procedure may be distorted, but its strength is that it elicits information from everyone, even people who are completely biased. As $n$ increases, this strength increases. When there are no partisans at all, of course majority rule is superior, because everyone wants to report truthfully. When there are a small number of partisans, their information can be discarded with little loss of welfare. But for example when $n=101$, even if there are as few as 12 partisans ( 6 on each side) then the SP procedure is better than majority rule.

The second way to approach this issue is to drop the assumption that everyone's evidence matters equally, and consider all possible procedures, not just anonymous ones. The mechanism design approach can then find the best possible equilibrium of the best possible procedure (see for example Myerson, 1991). In our earlier example in which 
$n=3$ and $\phi_{1}(a)=3 / 4, \phi_{2}(a)=1 / 4$, and $\phi_{3}(a)=1 / 2$, the best possible procedure for the unbiased person 3 is the same as majority rule except that $f((a, b, b), a)=4 / 7$ and $f((a, b, a), b)=4 / 7$. In other words, person 1 , who is biased toward $a$, gets to "enforce" her favorite with probability $4 / 7$ even when everyone else votes for $b$, and similarly person 2 , who is biased toward $b$, gets to "enforce" her favorite with probability $4 / 7$ even when everyone else votes for $a$. Biased individuals are given "special powers" (as in Chwe, 1999). The expected utility of the unbiased person 3 given this optimal nonanonymous procedure, which is the highest expected utility that an unbiased person can attain in any equilibrium of any procedure, is $44 / 63 \approx 0.698$.

Finding optimal nonanonymous procedures is not mathematically difficult, but specifying them can require a great deal of detail, since they are defined on $2^{n}$ cases, and they also typically depend precisely on people's biases (see for example Chwe, 1999). With the simplifying assumption that everyone's evidence matters equally, at least we can write down a procedure which is simple, does not depend precisely on people's biases, is incentive compatible for all biases, and is optimal given our assumption.

This paper does not find the best possible equilibrium of the best possible anonymous procedure, because in the best possible equilibrium of an anonymous procedure, some people might report truthfully and some might not, and our assumption in this paper is that everyone's evidence matters equally. Perhaps it is not reasonable to ask that a procedure elicit truthful reports from everyone, including people who are strongly biased in opposite directions. From this point of view, what is interesting about the SP procedure is not so much its optimality but the fact that it even exists. It might seem difficult to get truthful revelation from both a person very biased toward $a$ and a person very biased toward $b$ without being able to tell the two apart, but it is possible.

The optimality result of Proposition 3 is from the point of view of a perfectly unbiased person, which happens to be the objective function assumed by Condorcet in his original model. Still, it is natural to ask whether the optimality of the SP procedure depends in a fragile way on this particular objective function. Numerical computations show that this is an issue only for $q$ near 0.5 . Figure 5 shows the region in which the SP procedure is optimal, in terms of $q$ and $\phi_{0}(a)$, where $n=9$. Figure 5 also shows the regions in which the trivial procedures $f_{A}$ and $f_{B}$ are optimal, where $f_{A}$ is the procedure which chooses $a$ with probability 1 regardless of the reports and $f_{B}$ is the procedure which chooses $b$ with probability 1 regardless of the reports. For $q$ close to 0.5 , the optimality of $f_{S P}$ is not very robust. This is partly because when the quality of evidence is very low, someone with even the slightest bias toward $a$ finds $f_{A}$ optimal and someone with even the slightest bias toward $b$ finds $f_{B}$ optimal. For $q$ greater than around $0.6, f_{S P}$ is optimal for people whose biases are in a significant interval around 0.5 . For $q$ greater than around $0.9, f_{S P}$ is optimal for almost all biases.

It is also natural to consider other welfare criteria such as total social welfare. Recall that average social welfare is equal to the utility of an individual whose bias is the average of biases in the group. Thus if biases have average $\phi(a)=\phi(b)=1 / 2$, then maximizing total social welfare is equivalent to maximizing the utility of an unbiased person. Similarly, maximizing the weighted sum of utilities is equivalent to maximizing the utility of an individual whose bias is the weighted average of biases. Thus in Figure 5, 


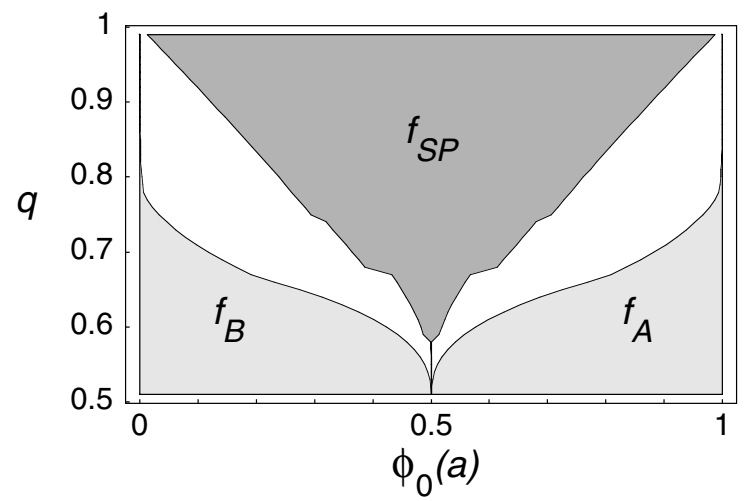

Figure 5. Anonymous incentive compatible procedures which maximize $E U_{0}$ given $\phi_{0}(a) \in[0,1]$ and $q=0.51,0.52, \ldots, 0.99$, where $n=9$.

for $q$ greater than around $0.9, f_{S P}$ is optimal for almost any weighted sum of individual utilities; in other words, most of the Pareto frontier is $f_{S P}$.

\section{CONCLUSION}

This paper starts with Condorcet's original model, adds strategic voting and heterogeneous prior beliefs and preferences, and finds an optimal and surprisingly robust nonmonotonic voting procedure. This robustness and nonmonotonicity is not due to any particular procedure, but is an "artifact" of Condorcet's model itself. Other extensions of Condorcet's model include giving each person a continuous, not binary, signal about which alternative is superior (for example Duggan and Martinelli, 2001; Li et al., 2001) and giving some people more informative signals than others (for example Ben-Yashar and Milchtaich, 2007). The assumption of binary signals in our paper greatly simplifies the consideration of anonymous procedures. When signals are binary, an anonymous procedure is simply a function of the total number of reports for $a$. With continuous signals, an anonymous procedure is a function of everyone's continuous signals which is symmetric in its arguments, a much more complicated mathematical object. If we allow continuous signals, we might get similar results: for example, in a two-person model with continuous signals, one can show that a monotonic procedure cannot be incentive compatible (Li et al., 2001). If people have different quality evidence, which we would represent in our model by letting each person have a different value of $q$, then of course the results here would not hold; the SP procedure does depend on the specific value of $q$ (in fact $q$ and $n$ are the only parameters the SP procedure does depend on). Finding optimal incentive compatible procedures when signals are continuous or when people have different quality evidence is a question for future work. Another line of research explicitly models uncertainty in people's biases; it 
is possible that uncertainty about biases can help in getting people to truthfully reveal their evidence (Austen-Smith and Feddersen, 2006). Since the SP procedure is incentive compatible for all biases, it provides a minimum level of performance when biases are uncertain.

Despite its power, surprisingly few papers have used the mechanism design approach to analyze Condorcet's model (examples are Chwe, 1999; Li et al., 2001; Wolinsky, 2002). The mechanism design approach has several substantive advantages over starting with a single particular procedure like majority rule or unanimity rule. For example, since in real life people discuss and argue before voting, "pre-play communication" should be considered. But allowing this changes the strategic situation dramatically (Coughlan, 2000). In fact, if pre-play communication is unrestricted, then it really doesn't matter what the voting rule is: all voting rules except for unanimity rules generate the same set of equilibrium outcomes (Gerardi and Yariv, 2007). Thus pre-play communication should be considered an integral part of the procedure itself. Since there are many possible kinds of pre-play communications, including bilateral conversations, group announcements, and straw polls, it might seem almost impossible to find which procedure is socially optimal. But the mechanism design approach does exactly this. The mechanism design approach asks the more profound question of what is the best possible procedure, not what behavior is given a particular procedure. But it is mathematically simpler, just a linear programming problem.

Most of the existing work on Condorcet's model is "conservative" in that it considers already well-known procedures such as majority rule and unanimity rule. But the entire point of Condorcet's original argument is to derive the optimal procedure, not assume it. When we add strategic voting, mechanism design allows us to keep the spirit of Condorcet's original question. This paper shows that if we assume strategic voting and anonymity in the sense of everyone's evidence affecting the decision equally, Condorcet's model cannot be understood as supporting well-known procedures such as majority rule as long as there is at least one person biased in each direction. The SP procedure is the optimal anonymous procedure for a very large set of biases (when there is at least one person biased in each direction) and is incentive compatible for all biases. The SP procedure is not monotonic, but no incentive compatible anonymous procedure is monotonic when there is at least one person biased in each direction.

Would anyone actually use a nonmonotonic procedure in practice? Nonmonotonic procedures are not that unusual. In a similar model in which experts who all have similar biases report to a decision maker with a different bias, the optimal procedure for the decision maker is nonmonotonic (Wolinsky, 2002). When deciding among more than two alternatives, some popular procedures are nonmonotonic, such as plurality voting with a runoff among the top two candidates (Riker, 1982; but see also Austen-Smith and Banks, 1991). If one is wedded to monotonicity, the results here might be understood as showing the limitations of procedures which treat everyone's evidence equally: given biases in both directions, one cannot retain monotonicity without treating some people's evidence differently from others. Perhaps our results illustrate the limitations of Condorcet's model itself; although it is often used as a foundation for modeling voting and decision-making, it can yield surprisingly nonintuitive results. 


\section{APPENDIX}

Lemma 1 Say that $f$ is anonymous and symmetric. Then person i's two incentive compatibility constraints can be written as:

$$
\begin{aligned}
& \left(\phi_{i}(a)-q\right) W(q, \gamma) \geq 0 \\
& \left(\phi_{i}(b)-q\right) W(q, \gamma) \geq 0,
\end{aligned}
$$

where $\gamma(\alpha(r))=f(r, a)$ and $W(q, \gamma)=\sum_{j=0}^{n-1} C_{j}^{n-1} q^{j}(1-q)^{n-1-j}(\gamma(j)-\gamma(j+1))$, and $C_{j}^{n}$ is the binomial coefficient $C_{j}^{n}=n ! /((n-j) ! j !)$.

Proof: Since we consider anonymous procedures $f$, we write $f(r, a)=\gamma(\alpha(r))$, where $\gamma(j)$ is the probability that the procedure chooses $a$ given that there are $j$ reports of $a$.

Let $A(\phi, \gamma)=E U_{i}\left(f, i d, i d_{-i}\right)-E U_{i}\left(f, s_{a a}, i d_{-i}\right)$ be person $i$ 's utility difference between playing $i d$ and $s_{a a}$ given that everyone else plays $i d$. Similarly, let $B(\phi, \gamma)=E U_{i}\left(f, i d, i d_{-i}\right)-E U_{i}\left(f, s_{b b}, i d_{-i}\right)$. Person $i$ 's incentive compatibility constraints are $A(\phi, \gamma) \geq 0$ and $B(\phi, \gamma) \geq 0$. By our formula $(*)$, we have $A(\phi, \gamma)=$ $\phi(a)\left[p_{a a}\left(f, i d, i d_{-i}\right)-p_{a a}\left(f, s_{a a}, i d_{-i}\right)\right]+\phi(b)\left[p_{b b}\left(f, i d_{,}, i d_{-i}\right)-p_{b b}\left(f, s_{a a}, i d_{-i}\right)\right]$.

Show that $p_{a a}\left(f, i d_{,}, d_{-i}\right)-p_{a a}\left(f, s_{a a}, i d_{-i}\right)=(1-q) \sum_{j=0}^{n-1} C_{j}^{n-1} q^{j}(1-q)^{n-1-j}(\gamma(j)-$ $\gamma(j+1))=(1-q) W(q, \gamma)$. To see this fact, note that $p_{a a}\left(f, i d, i d_{-i}\right)$ and $p_{a a}\left(f, s_{a a}, i d_{-i}\right)$ are different only when $e_{i}=b$ (when $e_{i}=a$, in both $i d$ and $s_{a a}$, person $i$ makes the same report). Since the superior alternative is $a$, the probability that $e_{i}=b$ is $1-q$. The probability that $j$ other people have evidence $a$ is $C_{j}^{n-1} q^{j}(1-q)^{n-1-j}$. If person $i$ plays $i d$, then the group chooses $a$ with probability $\gamma(j)$; if person $i$ plays $s_{a a}$, then the group chooses $a$ with probability $\gamma(j+1)$; thus the fact is demonstrated. Similarly, we find that $p_{b b}\left(f, i d, i d_{-i}\right)-p_{b b}\left(f, s_{a a}, i d_{-i}\right)=q \sum_{j=0}^{n-1} C_{j}^{n-1}(1-q)^{j} q^{n-1-j}(1-\gamma(j)-(1-$ $\gamma(j+1)))=q \sum_{j=0}^{n-1} C_{j}^{n-1}(1-q)^{j} q^{n-1-j}(\gamma(j+1)-\gamma(j))$. If we change variables and let $i=n-1-j$, this is equal to $q \sum_{i=0}^{n-1} C_{n-1-i}^{n-1}(1-q)^{n-1-i} q^{i}(\gamma(n-i)-\gamma(n-1-i))$. Since $f$ is symmetric, we have $\gamma(n-i)-\gamma(n-1-i)=1-\gamma(i)-(1-\gamma(i+1))=\gamma(i+1)-\gamma(i)$. We also have $C_{n-1-i}^{n-1}=C_{i}^{n-1}$. Hence $p_{b b}\left(f, i d_{,}, i d_{-i}\right)-p_{b b}\left(f, s_{a a}, i d_{-i}\right)=-q W(q, \gamma)$.

Thus $A(\phi, \gamma)=\phi(a)(1-q) W(q, \gamma)-\phi(b) q W(q, \gamma)=(\phi(a)-q \phi(a)-(1-$ $\phi(a)) q) W(q, \gamma)=(\phi(a)-q) W(q, \gamma)$. We similarly show $B(\phi, \gamma)=(\phi(b)-q)$ $W(q, \gamma)$.

Proposition 1 Say that there exist $i, j \in N$ such that $\phi_{i}(b)>q$ and $\phi_{j}(a)>q$. Iff is anonymous and incentive compatible for persons $i$ and $j$, then person l's incentive compatibility constraints hold with equality for any $\phi_{l}$.

Proof: Let $r=\min \left\{\phi_{i}(b), \phi_{j}(a)\right\}$. We know $r>q$. Define biases $\phi_{r}$ and $\phi_{1-r}$ as $\phi_{r}(a)=r, \phi_{r}(b)=1-r$ and $\phi_{1-r}(a)=1-r, \phi_{1-r}(b)=r$. It is easy to see that $\phi_{r}(a), \phi_{1-r}(a) \in\left[\phi_{i}(a), \phi_{j}(a)\right]$. Since $f$ satisfies $A\left(\phi_{i}, \gamma\right) \geq 0, B\left(\phi_{i}, \gamma\right) \geq 0, A\left(\phi_{j}, \gamma\right) \geq$ $0, B\left(\phi_{j}, \gamma\right) \geq 0$, since $A(\phi, \gamma)$ and $B(\phi, \gamma)$ are linear in $\phi(a)$, and $\phi_{r}(a), \phi_{1-r}(a) \in$ $\left[\phi_{i}(a), \phi_{j}(a)\right]$, we know $f$ satisfies $A\left(\phi_{r}, \gamma\right) \geq 0, B\left(\phi_{r}, \gamma\right) \geq 0$ and $A\left(\phi_{1-r}, \gamma\right) \geq 0$, $B\left(\phi_{1-r}, \gamma\right) \geq 0$. 
Define $\gamma^{\prime \prime}(j)=1-\gamma(n-j)$. It is easy to verify (by simply exchanging the names of $a$ and $b$ ) that $A\left(\phi_{r}, \gamma^{\prime \prime}\right)=B\left(\phi_{1-r}, \gamma\right) \geq 0$ and $A\left(\phi_{1-r}, \gamma^{\prime \prime}\right)=B\left(\phi_{r}, \gamma\right) \geq 0$. Define $\gamma^{\prime}(j)=\left(\gamma(j)+\gamma^{\prime \prime}(j)\right) / 2$. Now $A\left(\phi_{r}, \gamma^{\prime}\right)=\left(A\left(\phi_{r}, \gamma\right)+A\left(\phi_{r}, \gamma^{\prime \prime}\right)\right) / 2 \geq 0$ and $A\left(\phi_{1-r}, \gamma^{\prime}\right)=\left(A\left(\phi_{1-r}, \gamma\right)+A\left(\phi_{1-r}, \gamma^{\prime \prime}\right)\right) / 2 \geq 0$. It is easy to see that $\gamma^{\prime}(j)$ is symmetric and thus as shown in the proof of Lemma 1, we have $A\left(\phi_{r}, \gamma^{\prime}\right)=(r-q) W\left(q, \gamma^{\prime}\right)$ and $A\left(\phi_{1-r}, \gamma^{\prime}\right)=(1-r-q) W\left(q, \gamma^{\prime}\right)$. Hence $(r-q) W\left(q, \gamma^{\prime}\right) \geq 0$ and $(1-r-q) W\left(q, \gamma^{\prime}\right) \geq 0$. Since $r-q>0$ and $1-r-q<0$, we have $W\left(q, \gamma^{\prime}\right)=0$. Hence $A\left(\phi_{r}, \gamma^{\prime}\right)=0$. But since $A\left(\phi_{r}, \gamma\right) \geq 0$ and $A\left(\phi_{r}, \gamma^{\prime \prime}\right) \geq 0$, and $A\left(\phi_{r}, \gamma^{\prime}\right)=\left(A\left(\phi_{r}, \gamma\right)+A\left(\phi_{r}, \gamma^{\prime \prime}\right)\right) / 2$, we have $A\left(\phi_{r}, \gamma\right)=A\left(\phi_{r}, \gamma^{\prime \prime}\right)=0$. Since $B\left(\phi_{1-r}, \gamma\right)=A\left(\phi_{r}, \gamma^{\prime \prime}\right)$, we have $B\left(\phi_{1-r}, \gamma\right)=0$. Similarly, we conclude that $A\left(\phi_{1-r}, \gamma\right)=0$ and $B\left(\phi_{r}, \gamma\right)=0$.

Now for any $\phi_{l}$, we have $\phi_{l}(a)=\lambda \phi_{r}(a)+(1-\lambda) \phi_{1-r}(a)$, where $\lambda \in \Re$ (that is, $\phi_{l}(a)$ is a linear, not necessarily convex, combination of $\phi_{r}(a)$ and $\left.\phi_{1-r}(a)\right)$, and thus $A\left(\phi_{l}, \gamma\right)=\lambda A\left(\phi_{r}, \gamma\right)+(1-\lambda) A\left(\phi_{1-r}, \gamma\right)=0$ and similarly $B\left(\phi_{l}, \gamma\right)=0$.

Proposition 2 Say that there exist $i, j \in N$ such that $\phi_{i}(b)>q$ and $\phi_{j}(a)>q$. Say $f$ is an anonymous incentive compatible procedure which is not trivial. Then $f$ is not monotonic.

Proof: Say $f$ is anonymous, incentive compatible, and not trivial. Let $\gamma(\alpha(r))=f(r, a)$. Say $f$ is monotonic, in other words $\gamma(j) \leq \gamma(j+1)$ for all $j$. As in the proof of Proposition 1 , define $\gamma^{\prime \prime}(j)=1-\gamma(n-j)$ and define $\gamma^{\prime}(j)=\left(\gamma(j)+\gamma^{\prime \prime}(j)\right) / 2$. Note that $\gamma^{\prime}$ is symmetric. It is easy to see that $\gamma^{\prime}$ is monotonic: since $\gamma(n-j) \geq \gamma(n-j-1)$, we have $1-\gamma(n-j) \leq 1-\gamma(n-j-1)$ and thus $\gamma^{\prime \prime}(j) \leq \gamma^{\prime \prime}(j+1)$, and since $\gamma(j) \leq \gamma(j+1)$, we have $\gamma^{\prime}(j) \leq \gamma^{\prime}(j+1)$. Since $f$ is not trivial, there exists $k$ such that $\gamma(k)<\gamma(k+1)$. Since $f$ is monotonic, we have $\gamma(n-k-1) \leq \gamma(n-k)$ and thus $\gamma^{\prime \prime}(k) \leq \gamma^{\prime \prime}(k+1)$. Therefore, $\gamma^{\prime}(k)<\gamma^{\prime}(k+1)$.

The proof of Proposition 1 shows that $W\left(q, \gamma^{\prime}\right)=0$. But $W\left(q, \gamma^{\prime}\right)$ is the weighted sum of $\gamma^{\prime}(j)-\gamma^{\prime}(j+1)$ terms, where all of the weights are greater than zero. Since $\gamma^{\prime}(j)-\gamma^{\prime}(j+1) \leq 0$ for all $j$, it must be that $\gamma^{\prime}(j)-\gamma^{\prime}(j+1)=0$ for all $j$, which contradicts $\gamma^{\prime}(k)<\gamma^{\prime}(k+1)$.

Lemma 2 Given $q$ and $n$, there uniquely exists $k \in\{0,1, \ldots,(n-3) / 2\}$ such that

$$
z=\frac{D_{k} y^{(n-1) / 2}-k\left(y^{k-1}+y^{n-k}\right)}{(n-k)\left(y^{k}+y^{n-1-k}\right)-k\left(y^{k-1}+y^{n-k}\right)} \in(0,1],
$$

where $y=q /(1-q)$ and $D_{k}=(k !(n-k) !) /(((n-1) / 2) !)^{2}$. Also, $k$ is nonincreasing in $q$ and $k<(1-q) n$. As $n$ grows large, $k / n \rightarrow \rho$, where $\rho<1-q$ is uniquely defined by $\rho \log \rho+(1-\rho) \log (1-\rho)+\log 2=(1 / 2-\rho) \log y$.

Proof: We write $z$ as a function of $k$ and $y$ and multiply numerator and denominator by $y^{-(n-1) / 2}$ to get $z_{k}(y)=\operatorname{num}_{k}(y) / \operatorname{den}_{k}(y)$, where $\operatorname{num}_{k}(y)$ and $\operatorname{den}_{k}(y)$ are defined as:

$$
\begin{gathered}
\operatorname{num}_{k}(y)=D_{k}-k\left(y^{k-n / 2-1 / 2}+y^{n / 2-k+1 / 2}\right) \\
\operatorname{den}_{k}(y)=(n-k)\left(y^{k-n / 2+1 / 2}+y^{n / 2-k-1 / 2}\right)-k\left(y^{k-n / 2-1 / 2}+y^{n / 2-k+1 / 2}\right) .
\end{gathered}
$$


Note that $\operatorname{num}_{k}(y)$ is strictly decreasing in $y$ for $y>1$ and $k \in\{1, \ldots,(n-3) / 2\}$, because $(d / d y)\left(\operatorname{num}_{k}(y)\right)=-(k / y)(n / 2-k+1 / 2)\left(-y^{k-n / 2-1 / 2}+y^{n / 2-k+1 / 2}\right)<0$.

We prove five useful facts. Fact A is $\operatorname{num}_{k+1}(y) \leq 0 \Leftrightarrow \operatorname{num}_{k}(y) \leq \operatorname{den}_{k}(y)$. This is because $\operatorname{num}_{k+1}(y) \leq 0$ is equivalent to $D_{k+1} /(k+1) \leq y^{k-n / 2+1 / 2}+y^{n / 2-k-1 / 2}$ and $\operatorname{num}_{k}(y) \leq \operatorname{den}_{k}(y)$ is equivalent to $D_{k} /(n-k) \leq y^{k-n / 2+1 / 2}+y^{n / 2-k-1 / 2}$, and it is easy to verify from the definition of $D_{k}$ that $D_{k+1} /(k+1)=D_{k} /(n-k)$. Similarly, we have Fact B: $\operatorname{num}_{k+1}(y)>0$ is equivalent to $\operatorname{num}_{k}(y)>\operatorname{den}_{k}(y)$.

Fact $\mathrm{C}$ is that $\operatorname{num}_{k}(y) \geq 0$ is equivalent to

$$
\frac{n-k}{k} \cdot \frac{n-k-1}{k+1} \cdots \frac{(n+1) / 2}{(n-1) / 2} \geq y^{k-n / 2-1 / 2}+y^{n / 2-k+1 / 2} .
$$

To see this, note that $\operatorname{num}_{k}(y) \geq 0$ is equivalent to $D_{k} / k \geq y^{k-n / 2-1 / 2}+y^{n / 2-k+1 / 2}$ and

$$
D_{k} / k=\frac{(n-k) !}{((n-1) / 2) !} \frac{(k-1) !}{((n-1) / 2) !}=\frac{n-k}{k} \cdot \frac{n-k-1}{k+1} \cdots \frac{(n+1) / 2}{(n-1) / 2} .
$$

Similarly, we have Fact D: $\operatorname{num}_{k}(y)>0$ is equivalent to

$$
\frac{n-k}{k} \cdot \frac{n-k-1}{k+1} \cdots \frac{(n+1) / 2}{(n-1) / 2}>y^{k-n / 2-1 / 2}+y^{n / 2-k+1 / 2} .
$$

Fact $\mathrm{E}$ is $\operatorname{num}_{k+1}(y) \geq 0 \Rightarrow \operatorname{num}_{k}(y)>0$ for $k \in\{0, \ldots,(n-3) / 2\}$. If $\operatorname{num}_{k+1}(y) \geq$ 0 , from Fact $\mathrm{C}$ we have

$$
\frac{n-k-1}{k+1} \cdot \frac{n-k-2}{k+2} \cdots \frac{(n+1) / 2}{(n-1) / 2}>y^{n / 2-k-1 / 2}
$$

since $y^{k-n / 2+1 / 2}>0$. Note that there are $n / 2-k-1 / 2$ terms on the left-hand side of this inequality which are all less than $(n-k) / k$. Hence $[(n-k) / k]^{n / 2-k-1 / 2}>y^{n / 2-k-1 / 2}$ and thus we get the inequality $(n-k) / k>y$. Fact $\mathrm{C}$ gives us the inequality

$$
\frac{n-k-1}{k+1} \cdot \frac{n-k-2}{k+2} \cdots \frac{(n+1) / 2}{(n-1) / 2} \geq y^{k-n / 2+1 / 2}+y^{n / 2-k-1 / 2} .
$$

We multiply these inequalities together and get

$$
\frac{n-k}{k} \cdot \frac{n-k-1}{k+1} \cdot \frac{n-k-2}{k+2} \cdots \frac{(n+1) / 2}{(n-1) / 2}>y^{k-n / 2+3 / 2}+y^{n / 2-k+1 / 2} .
$$

But $y^{k-n / 2+3 / 2}>y^{k-n / 2-1 / 2}$ since $y>1$ and thus by Fact $\mathrm{D}$ we have $\operatorname{num}_{k}(y)>0$.

Since $q \in(1 / 2,1)$, we have $y=q /(1-q) \in(1, \infty)$. Let $y \in(1, \infty)$ and show that there uniquely exists $k \in\{0,1, \ldots,(n-3) / 2\}$ such that $z_{k}(y) \in(0,1]$.

Note that $\operatorname{num}_{0}(y)=D_{0}>0$. Since $\operatorname{num}_{(n-1) / 2}(1)=D_{(n-1) / 2}-(n-1)=(n+1) / 2-$ $(n-1)=(3-n) / 2 \leq 0$ and $\operatorname{num}_{(n-1) / 2}(y)$ is strictly decreasing for $y>1$, we know 
$\operatorname{num}_{(n-1) / 2}(y)<0$. Hence there exists $k \in\{0, \ldots,(n-3) / 2\}$ such that $\operatorname{num}_{k}(y)>0$ and $\operatorname{num}_{k+1}(y) \leq 0$. Note that this $k$ is unique: say $\operatorname{num}_{k}(y)>0, \operatorname{num}_{k+1}(y) \leq 0$, $\operatorname{num}_{k^{\prime}}(y)>0$ and $\operatorname{num}_{k^{\prime}+1}(y) \leq 0$ for $k<k^{\prime}$. Then we have $\operatorname{num}_{k+1}(y) \leq 0$ and $\operatorname{num}_{k^{\prime}}(y)>0$, which contradicts Fact E.

Show $z_{k}(y) \in(0,1]$. Since $\operatorname{num}_{k+1}(y) \leq 0$, we know num $n_{k}(y) \leq \operatorname{den}_{k}(y)$ by Fact A. Since $\operatorname{num}_{k}(y)>0$, we know $z_{k}(y)=\operatorname{num}_{k}(y) / \operatorname{den}_{k}(y) \in(0,1]$.

Show $z_{j}(y) \notin(0,1]$ or is undefined for $j \in\{0, \ldots,(n-3) / 2\}, j \neq k$. Since $j \neq k$, we have three possible cases: (i) $\operatorname{num}_{j}(y)>0$ and $\operatorname{num}_{j+1}(y)>0$, (ii) num $\operatorname{nu}_{j}(y) \leq 0$ and $\operatorname{num}_{j+1}(y) \leq 0$, and (iii) num $m_{j}(y) \leq 0$ and $n u m_{j+1}(y)>0$. By Fact E, (iii) cannot happen. Also by Fact E, if $\operatorname{num}_{j+1}(y)=0$, then $\operatorname{num}_{j}(y) \leq 0$ is impossible and hence case (ii) reduces to $\operatorname{num}_{j}(y) \leq 0$ and $\operatorname{num}_{j+1}(y)<0$. In case $(i)$, we have $\operatorname{num}_{j}(y)>\operatorname{den}_{j}(y)$ by Fact B; since $\operatorname{num}_{j}(y)>0$, if $\operatorname{den}_{j}(y)>0$, then $z_{j}(y)>1$, if $\operatorname{den}_{j}(y)<0$, then $z_{j}(y)<0$, and if $\operatorname{den}_{j}(y)=0$, then $z_{j}(y)$ is undefined. In case $(i i)$, we have $\operatorname{num}_{j}(y)<\operatorname{den}_{j}(y)$ by Fact A; since $\operatorname{num}_{j}(y) \leq 0$, if $\operatorname{den}_{j}(y)>0$, then $z_{j}(y) \leq 0$, if $\operatorname{den}_{j}(y)<0$, then $z_{j}(y)>1$, and if $\operatorname{den}_{j}(y)=0$, then $z_{j}(y)$ is undefined.

To show that $k$ is nonincreasing in $q$, it suffices to show that $k$ is nonincreasing in $y$, since $y=q /(1-q)$. Let $y<y^{\prime}$. Remember that $k$ is chosen so that $\operatorname{num}_{k}(y)>0$ and $\operatorname{num}_{k+1}(y) \leq 0$. Choose $k^{\prime}$ so that $\operatorname{num}_{k^{\prime}}\left(y^{\prime}\right)>0$ and $\operatorname{num}_{k^{\prime}+1}\left(y^{\prime}\right) \leq 0$. We show $k \geq k^{\prime}$ by contradiction. Say that $k<k^{\prime}$. Since $\operatorname{num}_{k^{\prime}}\left(y^{\prime}\right)>0$ and $k+1 \leq k^{\prime}$, by Fact E we have $\operatorname{num}_{k+1}\left(y^{\prime}\right)>0$. Hence by Fact $\mathrm{D}$ we have

$$
\frac{n-k-1}{k+1} \cdots \frac{(n+1) / 2}{(n-1) / 2}>\left(y^{\prime}\right)^{k-n / 2-1 / 2}+\left(y^{\prime}\right)^{n / 2-k+1 / 2} .
$$

But $\operatorname{num}_{k+1}(y) \leq 0$, and hence by Fact $\mathrm{D}$ we have

$$
\frac{n-k-1}{k+1} \cdots \frac{(n+1) / 2}{(n-1) / 2} \leq y^{k-n / 2-1 / 2}+y^{n / 2-k+1 / 2} .
$$

Thus we have $\left(y^{\prime}\right)^{k-n / 2-1 / 2}+\left(y^{\prime}\right)^{n / 2-k+1 / 2}<y^{k-n / 2-1 / 2}+y^{n / 2-k+1 / 2}$, which contradicts $y<y^{\prime}$ since $y, y^{\prime}>1$.

Since $\operatorname{num}_{k}(y)>0$, by the reasoning in the proof of Fact E, we have $(n-k) / k>y$ and thus $k<n /(y+1)=(1-q) n$ since $y=q /(1-q)$. To show $k / n \rightarrow \rho$ as $n \rightarrow \infty$, note that as $n$ grows large, $k$ is given by $\operatorname{num}_{k}(y)=0$, which is $(k-1) !(n-k) !=(((n-$ $1) / 2) !)^{2} y^{n / 2-k+1 / 2}$ as $n$ grows large. Using Stirling's approximation $\log m ! \approx m \log m-m$, we have

$$
\begin{aligned}
& (k-1) \log (k-1)+(n-k) \log (n-k) \\
& \quad=(n-1) \log ((n-1) / 2)+(n / 2-k+1 / 2) \log y .
\end{aligned}
$$

If we let $k=\rho n$, we get

$$
\begin{aligned}
& (\rho n-1) \log (\rho n-1)+(1-\rho) n \log ((1-\rho) n) \\
& \quad=(n-1) \log ((n-1) / 2)+(n / 2-\rho n+1 / 2) \log y .
\end{aligned}
$$


As $n$ grows large, we have $\rho n \log (\rho n)+(1-\rho) n \log ((1-\rho) n)=n \log (n / 2)+$ $n(1 / 2-\rho) \log y$, and thus $\rho \log (\rho n)+(1-\rho) \log ((1-\rho) n)=\log (n / 2)+(1 / 2-\rho) \log y$. We simplify to get $\rho \log \rho+(1-\rho) \log (1-\rho)+\log 2=(1 / 2-\rho) \log y$.

To show that this equation determines $\rho$ uniquely, write it as $h(\rho)=-\log 2$, where $h(x)=x \log x+(1-x) \log (1-x)-(1 / 2-x) \log y$ is defined on $(0,1)$. We can calculate $\partial^{2} h / \partial x^{2}=1 / x+1 /(1-x)>0$ for $x \in(0,1)$ and hence $h$ is strictly convex. Thus $h(x)=-\log 2$ for at most two values of $x$. Since $h(1 / 2)=-\log 2$, one of those values is $x=1 / 2$. Since $k / n \rightarrow \rho$, and $k / n<1-q<1 / 2$, we know that $\rho \neq 1 / 2$ and is thus defined uniquely. It is easy to show that $\partial h / \partial x=0$ at $x=1-q$, and hence if we have two solutions of $h(x)=-\log 2$, then one must be less than $1-q$ and one must be greater than $1-q$. Since the solution $x=1 / 2$ is greater than $1-q$, we have $\rho<1-q$.

Proposition 3 Say that there exist $i, j \in N$ such that $\phi_{i}(b)>q$ and $\phi_{j}(a)>q$. Then $f_{S P}$ is the anonymous incentive compatible procedure which uniquely maximizes $E U_{0}$, where $\phi_{0}(a)=\phi_{0}(b)=1 / 2$.

Proof: First we need three facts. Fact 1. If $y>1$ and $x>0$, then $y^{2 x}-x y^{x} \log$ $y-1>0$. Since this holds with equality when $x=0$, it suffices to show that $(d / d x)\left(y^{2 x}-x y^{x} \log y-1\right)=y^{x}\left(2 y^{x} \log y-\log y-x(\log y)^{2}\right)>0$, or in other words $2 y^{x} \log y-\log y-x(\log y)^{2}>0$. This is true when $x=0$; since $(d / d x)\left(2 y^{x} \log y-\right.$ $\left.\log y-x(\log y)^{2}\right)=(\log y)^{2}\left(2 y^{x}-1\right)>0$, we are done.

Fact 2. If $y>1$, then

$$
\theta(j)=\frac{y^{n-j}-y^{j}}{(n-j)\left(y^{j}+y^{n-1-j}\right)-j\left(y^{j-1}+y^{n-j}\right)}
$$

is positive and strictly increasing for $j \in[0, n / 2)$. Note that $\theta(0)=\left(y^{n}-1\right) /(n(1+$ $\left.\left.y^{n+1}\right)\right)>0$ since $y>1$. It suffices to show that $\partial \theta(j) / \partial j>0$ for $j \in[0, n / 2)$. We can write

$$
\theta(j)=\frac{y^{n}-y^{2 j}}{(n-j)\left(y^{2 j}+y^{n-1}\right)-j\left(y^{2 j-1}+y^{n}\right)} .
$$

After some computation, we find

$$
\frac{\partial \theta(j)}{\partial j}=\frac{(1+y) y^{4 j-1}\left(y^{2 n-4 j}-(2 n-4 j) y^{n-2 j}-1\right)}{\left((n-j)\left(y^{2 j}+y^{n-1}\right)-j\left(y^{2 j-1}+y^{n}\right)\right)^{2}},
$$

which has the same sign as $y^{2 n-4 j}-(2 n-4 j) y^{n-2 j}-1$. This is positive by Fact 1 (let $x=n-2 j>0)$.

Fact 3. If $s(j)>0$ and $r(j) / s(j)$ strictly increases in $j$, and $\sum_{j=0}^{m} s(j)>s>0$, then the constrained maximization problem

$$
x(0), \ldots, x(m) \in[0,1] \sum_{j=0}^{m}-r(j) x(j) \text { such that } \sum_{j=0}^{m} s(j) x(j)=s
$$


has a unique maximum in which $x(j)=1$ for $j<l, x(l) \in(0,1]$, and $x(j)=0$ for $j>l$, where $0 \leq l \leq m$.

To prove this, we write the Lagrangian

$$
L=\sum_{j=0}^{m}-r(j) x(j)+\lambda s(j) x(j)+(\mu(j)-v(j)) x(j) .
$$

Since we are maximizing a continuous function over a nonempty compact set, an optimum $x$ exists. By the Kuhn-Tucker theorem, there exists $\lambda \in \Re$ and $\mu(j), v(j) \geq 0$ such that $\partial L / \partial x(j)=0$, where $\mu(j)=0$ if $x(j)>0$ and $\nu(j)=0$ if $x(j)<1$. In other words, if we let

$$
M=\sum_{j=0}^{m}-r(j) x(j)+\lambda s(j) x(j)
$$

there exists $\lambda \in \Re$ such that $\partial M / \partial x(j) \geq 0$ if $x(j)>0, \partial M / \partial x(j) \leq 0$ if $x(j)<1$, and $\partial M / \partial x(j)=0$ if $x(j) \in(0,1)$. But $\partial M / \partial x(j)=-r(j)+\lambda s(j)=s(j)(-r(j) / s(j)+\lambda)$, and since $s(j)>0$, the sign of $\partial M / \partial x(j)$ is the sign of $-r(j) / s(j)+\lambda$. Since $-r(j) / s(j)$ strictly decreases in $j$, either $(i) \partial M / \partial x(j)>0$ for all $j$, (ii) $\partial M / \partial x(j)<0$ for all $j$, or (iii) there exists $l$, where $0 \leq l \leq m$, such that $\partial M / \partial x(j)>0$ for $j<l, \partial M / \partial x(l) \geq 0$, and $\partial M / \partial x(j)<0$ for $j>l$. If we have $(i)$, then $x(j)=1$ for all $j$, but this is not feasible since $\sum_{j=0}^{m} s(j)>s$. If we have $(i i)$, then $x(j)=0$ for all $j$, but this is not feasible since $s>0$. Hence we have (iii), and there exists $l$, where $0 \leq l \leq m$, such that $x(j)=1$ for $j<l, x(l) \in(0,1]$, and $x(j)=0$ for $j>l$. This maximum is unique because given that $x(j)=1$ for $j<l$ and $x(j)=0$ for $j>l$, a unique $x(l)$ satisfies the constraint $\sum_{j=0}^{m} s(j) x(j)=s$.

Now we start the main proof. Show that $f_{S P}$ is incentive compatible. Since $f_{S P}$ is symmetric, by Lemma 1 , it suffices to show that $W\left(q, \gamma_{S P}\right)=0$, where $\gamma_{S P}(\alpha(r))=$ $f_{S P}(r)$. It is easy to show this given the definition of $z$ and $k$ in Lemma 2.

Let $F$ be the set of anonymous incentive compatible procedures. Let $F^{\prime} \subset F$ be the set of symmetric anonymous incentive compatible procedures. First show that $f_{S P}$ uniquely maximizes $E U_{0}$ over $F^{\prime}$. By Lemma 1 , since $\phi_{i}(b)>q$, we know $f \in F^{\prime}$ is incentive compatible if and only if $W(q, \gamma)=0$. Hence we have the single constraint $W(q, \gamma)=0$, along with the constraints $\gamma(j) \in[0,1]$. Since $f$ is symmetric, our choice variables are $\gamma(0), \gamma(1), \ldots, \gamma((n-1) / 2)($ we have $\gamma((n+1) / 2)=$ $1-\gamma((n-1) / 2), \ldots, \gamma(n-1)=1-\gamma(1), \gamma(n)=1-\gamma(0))$. We can write the constraint $W(q, \gamma)=0$ in terms of these variables: $C_{(n-1) / 2}^{n-1} q^{(n-1) / 2}(1-q)^{(n-1) / 2}(2 \gamma((n-1) / 2)-1)+$ $\sum_{j=0}^{(n-3) / 2} C_{j}^{n-1}\left(q^{j}(1-q)^{n-1-j}+q^{n-j-1}(1-q)^{j}\right)(\gamma(j)-\gamma(j+1))=0$. If we let $y=q /(1-q)$ and multiply both sides by $(1-q)^{-n+1}$, we can write this constraint as simply $C_{(n-1) / 2}^{n-1} y^{(n-1) / 2}(2 \gamma((n-1) / 2)-1)+\sum_{j=0}^{(n-3) / 2} C_{j}^{n-1}\left(y^{j}+y^{n-1-j}\right)(\gamma(j)-\gamma(j+1))=0$, or in other words $2 C_{(n-1) / 2}^{n-1} y^{(n-1) / 2} \gamma((n-1) / 2)+\sum_{j=0}^{(n-3) / 2} C_{j}^{n-1}\left(y^{j}+y^{n-1-j}\right)(\gamma(j)-$ $\gamma(j+1))=C_{(n-1) / 2}^{n-1} y^{(n-1) / 2}$. 
Our objective function, the expected utility of a person with $\phi_{0}(a)=\phi_{0}(b)=1 / 2$, is

$$
\begin{aligned}
E U_{0} & =\phi_{0}(a) p_{a a}(f, i d, \ldots, i d)+\phi_{0}(b) p_{b b}(f, i d, \ldots, i d) \\
& =\phi_{0}(a) \sum_{j=0}^{n} C_{j}^{n} q^{j}(1-q)^{n-j} \gamma(j)+\phi_{0}(b) \sum_{j=0}^{n} C_{j}^{n}(1-q)^{j} q^{n-j}(1-\gamma(j)) \\
& =\frac{1}{2} \sum_{j=0}^{n} C_{j}^{n}(1-q)^{j} q^{n-j}+\frac{1}{2} \sum_{j=0}^{n} C_{j}^{n}\left[q^{j}(1-q)^{n-j}-(1-q)^{j} q^{n-j}\right] \gamma(j) .
\end{aligned}
$$

Since this first term is constant in $\gamma(j)$, maximizing $E U_{0}$ is equivalent to maximizing the second term. We can multiply the second term by the positive constant $2(1-q)^{-n}$ and thus we can write the objective as $\sum_{j=0}^{n} C_{j}^{n}\left(y^{j}-y^{n-j}\right) \gamma(j)$, where $y=q /(1-q)$. But

$$
\begin{aligned}
\sum_{j=0}^{n} C_{j}^{n}\left(y^{j}-y^{n-j}\right) \gamma(j) & =\sum_{j=0}^{(n-1) / 2} C_{j}^{n}\left(y^{j}-y^{n-j}\right) \gamma(j)+C_{n-j}^{n}\left(y^{n-j}-y^{j}\right) \gamma(n-j) \\
& =\sum_{j=0}^{(n-1) / 2} C_{j}^{n}\left(y^{j}-y^{n-j}\right) \gamma(j)+C_{n-j}^{n}\left(y^{n-j}-y^{j}\right)(1-\gamma(j)) \\
& =\sum_{j=0}^{(n-1) / 2} C_{n-j}^{n}\left(y^{n-j}-y^{j}\right)+2 \sum_{j=0}^{(n-1) / 2} C_{j}^{n}\left(y^{j}-y^{n-j}\right) \gamma(j)
\end{aligned}
$$

because $C_{n-j}^{n}=C_{j}^{n}$ and $\gamma(n-j)=1-\gamma(j)$ because $f$ is symmetric.

So our maximization problem is to choose $\gamma(0), \gamma(1), \ldots, \gamma((n-1) / 2) \in[0,1]$ to maximize $\sum_{j=0}^{(n-1) / 2} C_{j}^{n}\left(y^{j}-y^{n-j}\right) \gamma(j)$ such that $2 C_{(n-1) / 2}^{n-1} y^{(n-1) / 2} \gamma((n-1) / 2)+$ $\sum_{j=0}^{(n-3) / 2} C_{j}^{n-1}\left(y^{j}+y^{n-1-j}\right)(\gamma(j)-\gamma(j+1))=C_{(n-1) / 2}^{n-1} y^{(n-1) / 2}$. Let $r(j)=C_{j}^{n}\left(y^{n-j}-\right.$ $\left.y^{j}\right)$ be the coefficient on $\gamma(j)$ in the objective and let $s(j)=C_{j}^{n-1}\left(y^{j}+y^{n-1-j}\right)-$ $C_{j-1}^{n-1}\left(y^{j-1}+y^{n-j}\right)$ be the coefficient on $\gamma(j)$ in the constraint. Let $s=C_{(n-1) / 2}^{n-1} y^{(n-1) / 2}$. Then $r(j) / s(j)=n \theta(j)$, where $\theta(j)$ is defined in Fact 2 above. Fact 2 says that $\theta(j)$ is positive and strictly increasing. Since $\theta(j)$ is positive and $r(j)$ is positive, we know $s(j)$ is positive. Since we know that $\gamma_{S P}$ satisfies the constraint, we have $\sum_{j=0}^{(n-1) / 2} s(j)>s>0$. Thus we can use Fact 3 to conclude that there is a unique maximum $\gamma$, where $\gamma(j)=1$ for $j<l, \gamma(l) \in(0,1]$, and $\gamma(j)=0$ for $j>l$. By definition, also $\gamma_{S P}(j)=1$ for $j<k$, $\gamma_{S P}(k) \in(0,1]$, and $\gamma_{S P}(j)=0$ for $j>k$. Since both $\gamma$ and $\gamma_{S P}$ satisfy the constraint, it is easy to show that $k=l$ and hence $\gamma=\gamma_{S P}$. Thus $f_{S P}$ uniquely maximizes $E U_{0}$ over $F^{\prime}$.

Now show that $f_{S P}$ uniquely maximizes $E U_{0}$ over $F$. Say that $\gamma$ maximizes $E U_{0}$ over $F$. Given $\phi$, it suffices to show that $A\left(\phi, \gamma^{\prime \prime}\right)=0$ and $B\left(\phi, \gamma^{\prime \prime}\right)=0$. Define $\gamma^{\prime \prime}$ as $\gamma^{\prime \prime}(j)=1-\gamma(n-j)$ and show $\gamma^{\prime \prime}$ is incentive compatible. Define $\phi^{\prime \prime}$ as $\phi^{\prime \prime}(a)=\phi(b)$ and $\phi^{\prime \prime}(b)=\phi(a)$. Since $\gamma$ is incentive compatible, by Lemma 1 we have $A\left(\phi^{\prime \prime}, \gamma\right)=0$ and 
$B\left(\phi^{\prime \prime}, \gamma\right)=0$. But it is easy to show that $A\left(\phi, \gamma^{\prime \prime}\right)=B\left(\phi^{\prime \prime}, \gamma\right)$ and $B\left(\phi, \gamma^{\prime \prime}\right)=A\left(\phi^{\prime \prime}, \gamma\right)$, and hence $A\left(\phi, \gamma^{\prime \prime}\right)=0$ and $B\left(\phi, \gamma^{\prime \prime}\right)=0$.

Define $\gamma^{\prime}$ as $\gamma^{\prime}(j)=\left(\gamma(j)+\gamma^{\prime \prime}(j)\right) / 2$. Since $\gamma$ and $\gamma^{\prime \prime}$ are incentive compatible, by convexity, $\gamma^{\prime}$ is incentive compatible. It is easy to see that $E U_{0}$ given $\gamma$ is equal to $E U_{0}$ given $\gamma^{\prime \prime}$, and thus by convexity, $E U_{0}$ given $\gamma^{\prime}$ is equal to $E U_{0}$ given $\gamma$. Since $\gamma^{\prime}$ is symmetric and $\gamma$ maximizes $E U_{0}$ over $F, \gamma^{\prime}$ maximizes $E U_{0}$ over $F^{\prime}$. Since $\gamma_{S P}$ uniquely maximizes $E U_{0}$ over $F^{\prime}$, we have $\gamma^{\prime}=\gamma_{S P}$, in other words $(\gamma(j)+1-\gamma(n-j)) / 2=\gamma_{S P}(j)$. Show that $\gamma=\gamma_{S P}$. Note that for $j$ such that $\gamma_{S P}(j)=0$, we must have $\gamma(j)=0$ and $\gamma(n-j)=1$, and hence $\gamma_{S P}(j)=\gamma(j)$. For $j$ such that $\gamma_{S P}(j)=1$, we must have $\gamma(j)=1$ and $\gamma(n-j)=0$, and hence $\gamma_{S P}(j)=\gamma(j)$.

So the optimal $\gamma$ is uniquely determined except for $\gamma(k)$ and $\gamma(n-k)$. We show that $\gamma(k)$ and $\gamma(n-k)$ are uniquely determined. By Proposition 1, $\gamma$ satisfies the incentive compatibility constraints with equality for all $\phi$. Hence $A(\phi, \gamma)=0$, or in other words (as shown in the proof of Lemma 1), $\phi(a)(1-q) \sum_{j=0}^{n-1} C_{j}^{n-1} q^{j}(1-q)^{n-1-j}(\gamma(j)-\gamma(j+$ $1))+\phi(b) q \sum_{j=0}^{n-1} C_{j}^{n-1}(1-q)^{j} q^{n-1-j}(\gamma(j+1)-\gamma(j))=0$. Since this is true for $\phi(a)=$ $1, \phi(b)=0$ as well as for $\phi(a)=0, \phi(b)=1$, we have $\sum_{j=0}^{n-1} C_{j}^{n-1} q^{j}(1-q)^{n-1-j}(\gamma(j)-$ $\gamma(j+1))=0$ and $\sum_{j=0}^{n-1} C_{j}^{n-1}(1-q)^{j} q^{n-1-j}(\gamma(j+1)-\gamma(j))=0$. Since $\gamma(j)$ is uniquely determined except for $\gamma(k)$ and $\gamma(n-k)$, we have $\left((n-k) y^{k}-k y^{k-1}\right) \gamma(k)+\left(k y^{n-k}-(n-\right.$ $\left.k) y^{n-k-1}\right) \gamma(n-k)=v_{1}$ and $\left(k y^{n-k}-(n-k) y^{n-k-1}\right) \gamma(k)+\left((n-k) y^{k}-k y^{k-1}\right) \gamma(n-k)=$ $v_{2}$, where $v_{1}, v_{2}$ are constants in $\gamma(k), \gamma(n-k)$ and $y=q /(1-q)$. We have two linear equations in two unknowns, and thus to show that $\gamma(k), \gamma(n-k)$ are uniquely determined, it is sufficient to show that the determinant $\left((n-k) y^{k}-k y^{k-1}\right)^{2}-\left(k y^{n-k}-(n-k) y^{n-k-1}\right)^{2}$ is nonzero, or in other words $\left((n-k) y^{k}-k y^{k-1}\right)^{2} \neq\left(k y^{n-k}-(n-k) y^{n-k-1}\right)^{2}$. Since $n-k>k$ and $y>1$, we have $(n-k) y>k$ and thus $(n-k) y^{k}-k y^{k-1}>0$. Since from Lemma 2 we have $k y<n-k$, we have $k y^{n-k}-(n-k) y^{n-k-1}<0$. Thus the only way the determinant can be zero is if $(n-k) y^{k}-k y^{k-1}=-\left(k y^{n-k}-(n-k) y^{n-k-1}\right)$. If this is true, then $(n-k)\left(y^{k}-y^{n-k-1}\right)=k\left(y^{k-1}-y^{n-k}\right)$, which is impossible since $n-k>k$ and $y^{k}-y^{n-k-1}>y^{k-1}-y^{n-k}$. Since $\gamma(k), \gamma(n-k)$ are uniquely determined by the incentive compatibility constraints and $\gamma_{S P}(k), \gamma_{S P}(n-k)$ satisfy the incentive compatibility constraints, we have $\gamma(k)=\gamma_{S P}(k)$ and $\gamma(n-k)=\gamma_{S P}(n-k)$.

\section{REFERENCES}

Austen-Smith, David and Jeffrey Banks. 1991. "Monotonicity in Electoral Systems.” American Political Science Reviem 85: 531-537.

Austen-Smith, David and Timothy J. Feddersen. 2006. "Deliberation, Preference Uncertainty and Voting Rules." American Political Science Reviem 100: 209-217.

Ben-Yashar, Ruth and Igal Milchtaich. 2007. "First and Second Best Voting Rules in Committees." Social Choice and Welfare 29: 453-486.

Chwe, Michael Suk-Young. 1999. “Minority Voting Rights Can Maximize Majority Welfare.” American Political Science Reviem 93: 85-97.

Condorcet [Marie Jean Antoine Nicolas Caritat]. 1785. "Essai sur l'Application de l'Analyse à la Probabilité des Décisions Rendues à la Pluralité des Voix," Paris. 
Coughlan, Peter J. 2000. "In Defense of Unanimous Jury Verdicts: Mistrials, Communication, and Strategic Voting." American Political Science Reviem 94: 375-393.

Duggan, John and César Martinelli. 2001. "A Bayesian Model of Voting in Juries." Games and Economic Behavior 37: 259-294.

Feddersen, Timothy J. and Wolfgang Pesendorfer. 1996. "The Swing Voter's Curse." American Economic Reviem 86: 408-424.

Gerardi, Dino. 2000. “Jury Verdicts and Preference Diversity.” American Political Science Reviem 94: $395-406$.

Gerardi, Dino and Leeat Yariv. 2007. "Deliberative Voting." Fournal of Economic Theory 134: 317-338.

$\mathrm{Li}$, Hao, Sherwin Rosen, and Wing Suen. 2001. "Conflicts and Common Interests in Committees." American Economic Reviem 91: 1478-1497.

McLennan, Andrew. 1998. "Consequences of the Condorcet Jury Theorem for Beneficial Information Aggregation by Rational Agents." American Political Science Reviem 92: 413-418.

Myerson, Roger B. 1991. Game Theory: Analysis of Conflict. Cambridge, Massachusetts: Harvard University Press.

Riker, William H. 1982. Liberalism Against Populism: A Confrontation Between the Theory of Democracy and the Theory of Social Choice. San Francisco: W. H. Freeman.

Wolinsky, Asher. 2002. "Eliciting Information From Multiple Experts." Games and Economic Behavior 41: 141-160. 